Part of Journal of Research of the National Bureau of Standards, Volume 29, July 1942

\title{
A NEW DETERMINATION OF THE CONSTANT OF GRAVITATION
}

\author{
By Paul R. Heyl and Peter Chrzanowski
}

\section{ABSTRACT}

A new determination of the constant of gravitation by means of the torsion balance has been made in the hope of improving the precision of the result published in 1930. A number of suggested improvements in the apparatus were tried, and two of these were adopted The result obtained shows so slight an improvement over the 1930 result that it appears that the limiting point of diminishing returns has been reached with this form of apparatus.

\section{CONTENTS}

Introduction

II. Suggested improvements in the torsion balance

1. Best filament material

2. Length of filament

3. Increase inmass of attracting bodies

4. Multiple filament. 3

5. Bifilar suspension

6. Length of beam. 4

7. Increasing the number of masses

8. Disk beam

III. Adopted improvements_.

IV. Mathematical theory

V. Description of apparatus

VI. Method of observing

1. Adjustments

(a) Adjustment of cylinders

(b) Adjustment of pendulum

2. Length measurements

3. Time measurements _...

(a) Standard of time. 24

(b) Optical system 25

(c) The camera

VII. Results_...

\section{INTRODUCTION}

In $1930^{1}$ the senior author of the present paper published a redetermination of the constant of gravitation, the results of which, though of a precision higher than that of the previously accepted value, showed a curious relation among themselves for which no explanation was available. The work was done with the torsion balance, using small moving masses of gold, platinum, and glass. The results with each material were reasonably concordant among themselves, but there appeared to be considerable difference with the nature of the material. For instance, the mean values of the platinum and glass sets differed by an amount five times as great as the average departure from the mean in either set. Moreover, there was no overlap in the spreads of the two sets. While it seemed reasonable to attribute this

\footnotetext{
1 Paul R. Heyl, Redetermination of the constant of gravitation, BS J. Research 5, 1243 (1930) RP256.
} 
to experimental error of some nature, it was possible to interpret it as a departure from Newton's law of the constant proportionality of mass and weight. To test this point experiments were made with a Eötvös balance, using one ball of platinum and one of glass. The results were negative.

As stated in the 1930 paper, it was felt that the solution of this difficulty could best be attained by working with an apparatus sufficiently sensitive to give the next decimal place. The attempt to do this forms the subject matter of the present paper.

The general question of improvements in the torsion balance was considered, and a few suggestions that seemed promising were tried experimentally. Most of these, however, were failures, only two of them qualifying for adoption. For the benefit of future workers it has been thought well to discuss these failures in some detail.

\section{SUGGESTED IMPROVEMENTS IN THE TORSION BALANCE}

In the way in which the torsion balance was used, in the 1930 paper and in the present work, the time of swing was measured in two positions of the large attracting masses, called "near" and "far" (fig. 1). As compared with the free time of swing, with the large masses removed to a distance, the period in the near position will be shortened, and in the far position lengthened. The difference of these two times is a multiplying factor in the formula for the gravitational constant, and anything which will increase this difference without introducing other difficulties will be a move in the right direction. There are two ways in which this difference may be increased, by reducing the torsion of the suspending filament, and by increasing the moment of the force exerted by the large attracting masses upon the moving system. The latter may again be done in two ways, by increasing the force of attraction or by increasing the lever arm by which this force acts. We shall now consider a number of suggestions that come under these headings.

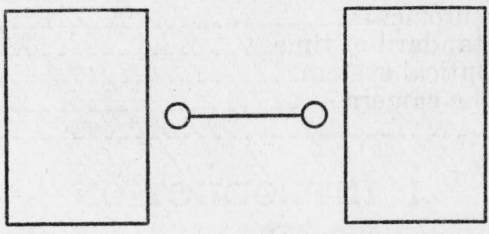

NEAR

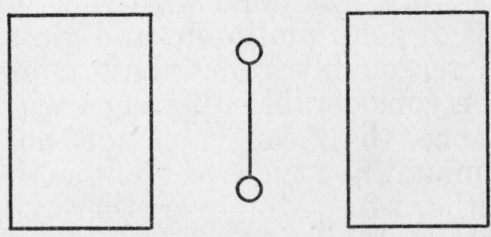

FAR

FIGURE 1.-Relative position of balls and cylinders. 


\section{BEST FILAMENT MATERIAL}

Since the modulus of torsion of a wire is proportional to the fourth power of its diameter, it is desirable to use a wire of as small a diameter as will safely carry the load of the beam and balls. This requires a material of high tensile strength. In this respect tungsten has no superior. Its tensile strength in the form of lamp filament 0.001 inch in diameter is, when hard drawn, from 300 to $400 \mathrm{~kg} / \mathrm{mm}^{2}$, and when annealed about 200. Larger sizes of tungsten filament show a somewhat smaller tensile strength, as this property increases with swaging and drawing. In comparison, the maximum value for chrome-vanadium steel is 232 , and for fused silica, 120 . In addition, tungsten can readily be obtained in finer wires than steel, and, as was found in the 1930 work, is the equal of fused silica in returning to the zero point after a large deflection.

\section{LENGTH OF FILAMENT}

The longer the filament the less the torsion for the same twist; but in practice there is a limit to the length that can safely be used. The filament is contained in a long tube rising from the lid of the apparatus case, and a slight warping at the bottom of the tube may be multiplied at the top to an amount sufficient to disturb seriously the centering adjustment of the beam. In practice, we have found that 1 meter is a safe limit to the length of the filament.

\section{INCREASE IN MASS OF ATTRACTING BODIES}

An increased mass of either the large or the small bodies will augment the force of attraction between them and heighten the sensitivity of the apparatus. With the small masses a limit of size is soon reached, as a filament of large diameter is required to carry the increased load, and by the fourth-power law the torsion will rapidly outrun the attraction.

There is no theoretical objection to increasing the large masses if material of sufficient homogeneity can be obtained in large pieces. Uniformity of density is important, as otherwise the position of the center of attraction would be uncertain.

\section{MULTIPLE FILAMENT}

It is theoretically possible to turn the fourth-power law of torsion to advantage. Suppose that a given load can be carried safely by a filament of diameter $D$ and torsion $\tau$. Replace this filament by two filaments of diameter $D / 2^{1 / 2}$ and torsion $\tau / 4$. The joint tensile strength of the double filament will be the same as that of the larger single filament, but the joint torsion will be half the previous value; and for more than two filaments the torsion will be rapidly reduced.

With a multiple filament of this character the question of friction between the components must be considered. We thought it worthwhile to test this experimentally, but an unforeseen practical difficulty prevented our getting any evidence on this point. This was the difficulty of obtaining an equal division of the load among the component filaments. Several attempts were made, but in every case the multiple filament broke down after a few days in operation. 


\section{BIFILAR SUSPENSION}

Theoretically there should be no difficulty in obtaining an equal division of load in the case of a bifilar suspension, and with a small separation between the two components the question of friction will not arise. However, in such a suspension the restoring force consists of both gravity and torsion, the gravity component varying as the square of the distance separating the component filaments, and this distance must therefore be kept small to avoid undue loss of sensitivity. But even if this distance be only a few tenths of a millimeter, the restoring force of gravity will be greater than that of torsion. This, however, appeared to be not without advantage. Experience with a single filament suspension indicated that the modulus of torsion, like most mechanical properties, varied slightly from time to time, probably from fatigue. Gravity; however, is constant under all known conditions, and in a suspension in which gravity is predominant in the restoring force, it seemed that greater uniformity in the observations might outweigh the disadvantage of a reduced time of swing.

A suspension was constructed with a separation of $0.5 \mathrm{~mm}$ between the filaments, maintained by the use of wire hooks at each end. At the top, each end of the filament was given a complete turn around the hook, and the loose ends were wrapped several times around the shank of the hook and fastened with hard wax. The hook at the lower end was passed through the loop of filament without wax or solder. Our experience with breakdowns in multiple filaments had impressed upon us the necessity of having the tension equal in both components, and this is difficult to obtain or to maintain when solder is used. It was thought that in a short time under load the tungsten wire would cut into the hook sufficiently to prevent slipping.

Measurements of time of swing with this suspension over a period of a month were disappointing. Fluctuations from day to day were observed, amounting to as much as 1 part in 3,000, probably due to slight shifts in the position of the filament on the lower hook. The time of swing varies inversely as the distance between the filaments, and when this distance is $0.5 \mathrm{~mm}$ a change in the time of 1 part in 3,000 would be produced by an alteration in the distance of 0.00017 $\mathrm{mm}$. If the separation were increased, this fluctuation would be reduced, but at the expense of a corresponding reduction in time of swing and diminished sensitivity of the apparatus.

\section{LENGTH OF BEAM}

The beam used in the 1930 apparatus was about $20 \mathrm{~cm}$ long. Considerable time was spent in the recent work in the attempt to use a beam $80 \mathrm{~cm}$ long. The difficulty with a beam of this length is its tendency to bend slightly with the weight of the balls during a long series of measurements, and thus alter the distance between the centers of the balls. Beams of different materials and different shapes of cross section were tried without overcoming this difficulty. The addition of braces to the beam to prevent this bending greatly increases the labor of computing the attraction of the large masses for the beam. This attraction, though only about 1 percent of the attraction for the balls, takes much longer to calculate.

Theoretically, it is possible to eliminate the attraction for the beam. Let the time of swing be measured with heavy balls on the beam. 
Replace these balls by lighter ones, and repeat the measurement of the time of swing. From two such measurements in both near and far positions it is possible to eliminate the attraction for the beam, other things remaining equal. But here comes in the difficulty. Other things (torsion, for instance) will not remain equal.

In order to change the balls it is necessary to relieve the filament temporarily of its load. The effect of this upon the torsion was experimentally investigated.

A series of 10 measurements of time of swing with a beam carrying platinum balls weighing $175 \mathrm{~g}$ each gave a mean value of 1892.62 $\pm 0.21 \mathrm{sec}$. The case was then opened and the beam let down on two supporting blocks. No change was made in the balls or in their position on the beam. The next day the beam was again raised and the case closed. The time of swing was then found to be 1886.69 sec. And since a change of balls could not be made without temporarily relieving the tension, the elimination of the attraction for the beam was found to be impracticable.

\section{INCREASING THE NUMBER OF MASSES}

By the use of a beam with four arms, carrying four balls, and providing four large masses, the total attraction can be doubled without increasing the load on any one arm of the beam. This arrangement, however, would be practicable only with rather long beams, which, as shown in the preceding section, are not practicable. A pair of large masses, when in the near position with respect to one pair of balls, will be in the far position with respect to the other pair, and these two effects will partly neutralize each other. This neutralization will be the more nearly complete as the arms of the beam are shortened. Carried to the limit, with arms so short that the large masses are close enough to touch each other, these masses would approximate a continuous ring, which would have no resultant attraction on the beam.

\section{DISK BEAM}

The idea of replacing the rod-shaped beam by a circular disk, carrying the balls at its circumference, is attractive from the point of view of the computer, since the torque of the large masses for such a disk is zero. The objection is the increase in weight. Such a disk, even though made of aluminum, would weigh more than the balls it carried, with the consequence of increased filament diameter and lessened sensitivity. Moreover, the moment of inertia of the disk would be great enough to be an important factor in the time of swing, and the differential effect of the large masses on the balls in the near and far positions would be correspondingly reduced.

\section{ADOPTED IMPROVEMENTS}

With the failure of the suggested improvements discussed in section II, it would appear that in dimensions, masses, and general arrangement the apparatus described in the 1930 paper is perhaps the best that is practicable. There were, however, two improvements which were found to be worthy of adoption. One of these was photographic recording, which will be described in detail in a later section. The other was a change in the position of the large attracting masses. 
In the 1930 apparatus the large masses were steel cylinders weighing about $66 \mathrm{~kg}$ each, arranged with their axes vertical. This position gave rise to two difficulties. In measuring the distance between the cylinders it was necessary to grope for the minimum distance. This required so many measurements that the whole operation normally required about $2 \frac{1}{2}$ days.

In addition, the time of swing in the near position varied considerably with the amplitude, making necessary an extrapolation for the limiting value of the time as the amplitude approached zero. In the far position the time-amplitude curve was nearly a straight horizontal line. The reason for this is obvious. With vertical cylinders in the near position, the balls swing in a gravitational field of varying in-

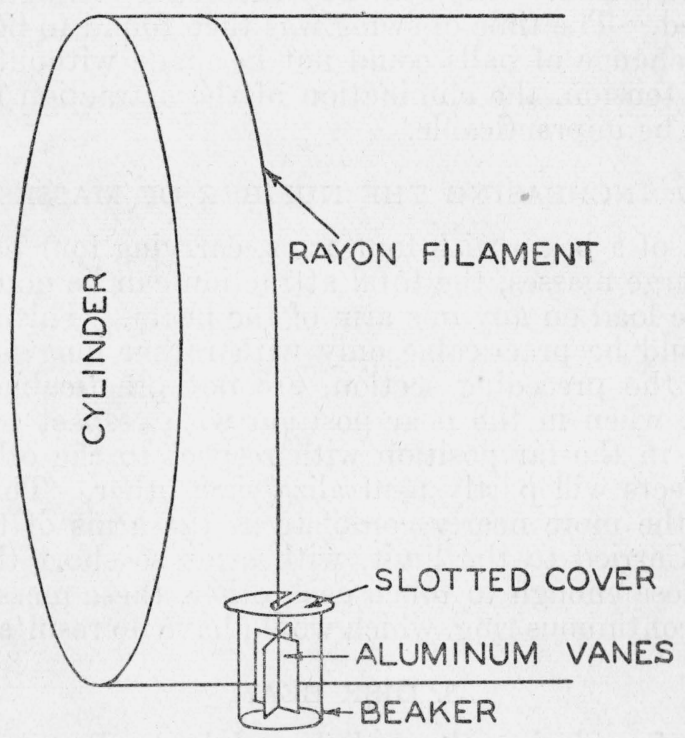

Figure 2.-Rayon plumb bob.

tensity, whereas in the far position the field is much more nearly uniform.

By laying the cylinders on their sides with their axes horizontal both these difficulties were avoided. In measuring the distance between the cylinders, delicate plumb bobs were hung touching the inner surface of each cylinder. This gave at once the minimum distance, which was measured by micrometer microscopes as described in the 1930 paper. These plumb bobs consisted of single filaments of rayon which were attached by a small fragment of adhesive tape to the upper surface of the cylinder near the ends, and hung down over the inner surfaces (fig. 2). The bob at the end of a filament was a small vane of sheet aluminum weighing about half a gram. To avoid disturbance from air currents this vane hung in a small empty beaker with a slotted cardboard cover. By this device the measurement of the distance between the cylinders could be made in about 3 hours.

After the measurements were made the beakers were removed, but the plumb bobs were left in place for use again after the time of swing had been measured. No correction was made for the attraction of 
the vanes on the moving system, as the effect of two vanes was of the order of one hundred thousandth of the attraction of the cylinder, and our results did not reach that degree of precision.

With the axes of the cylinders horizontal the time of swing in the near position became nearly independent of the amplitude, thus removing the uncertainty of the extrapolation. Near the middle of a cylinder the equipotential surfaces are very nearly parallel to the cylinder, and if the amplitude of the swing of the ball is not more than a few degrees, the motion takes place in a practically uniform gravitational field.

While this position of the cylinders simplifies the physical measurements, it greatly increases the labor of calculating the attraction of the cylinders on the pendulum. However, one must always use the simplest physical procedure, involving the least probability of experi-

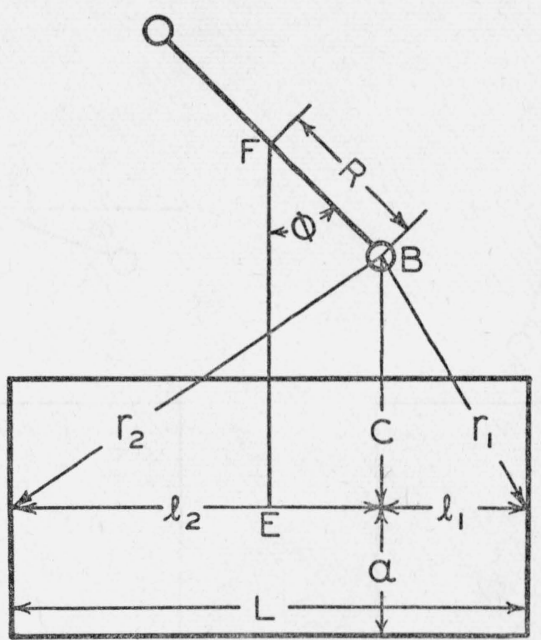

FIGURE 3.-Geometrical constants in attraction formula.

mental error, without shrinking from the mathematical labor which it may entail.

\section{MATHEMATICAL THEORY}

In deriving the formulas for the torsion pendulum as used in the present work, it will be convenient to consider first the action between a single cylinder and one ball of the pendulum (fig. 3). We shall assume for the present that the center of the ball is at the same level as the axis of the cylinder.

The beam of the pendulum is supported by a filament at $F$. From $F$ is drawn the line $F E$ perpendicular to the axis of the cylinder. The beam makes a position angle $\phi$ with $F E$, the positive direction of $\phi$ being counterclockwise. $B$ is the ball under consideration and $R$ its radius of gyration.

From $B$ draw a line of length $C$ perpendicular to the axis of the cylinder and dividing it into two parts, $l_{1}$ and $l_{2}$. In the present work, $l_{1}$ will be considered positive and $l_{2}$ negative, although in the 1930 paper both were regarded as positive. The distances from the ball $B$ to the ends of the axis of the cylinder are $r_{1}$ and $r_{2}$, respectively. 
It must be carefully noted that $\phi$ is merely an angle defining the position of the ball under consideration with respect to the cylinder which is attracting it, and does not necessarily measure the angle of displacement from the position of equilibrium, where the torque due to the suspending filament is zero. This angle of displacement will be denoted by $\theta$. Its positive direction is the same as that of $\phi$.

Figure. 4 shows the relation between $\phi$ and $\theta$ in the four cases that occur in practice. The relation may be summarized as follows:

Near position, close ball

Near position, distant ball $\phi=\theta$
$\phi=180^{\circ}+\theta$
$\phi=90^{\circ}+\theta$
$\phi=270^{\circ}+\theta$

In all cases it is to be noted that $d \phi=d \theta$ and $d^{2} \phi=d^{2} \theta$.
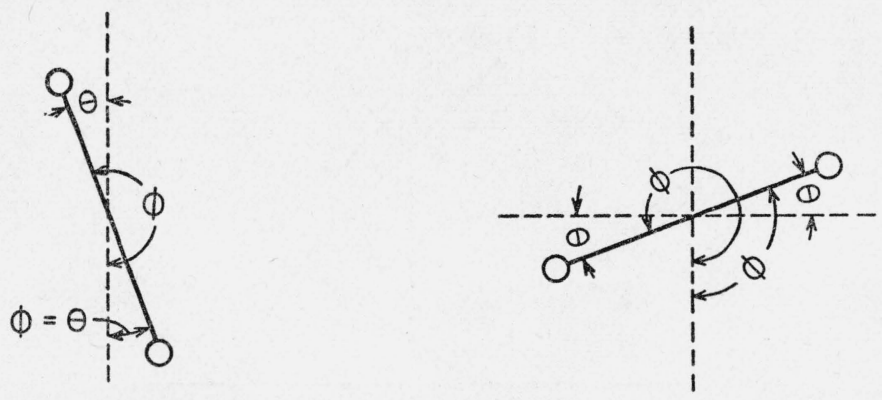

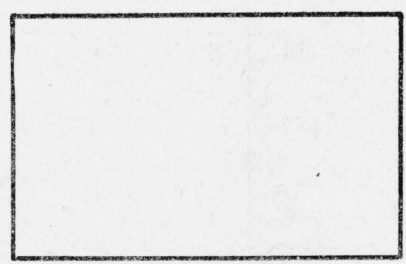

NEAR

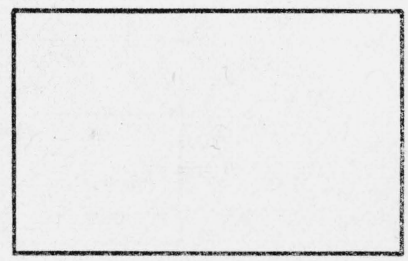

FAR

Figure 4.-Position and deflection angles.

The differential equation for the motion of the pendulum may be conveniently derived as an energy equation. Considering the complete system, beam, balls, and cylinders, for any position angle $\phi$ :

Kinetic energy of the pendulum + potential energy due to filament

tpotential energy of attraction $=$ constant.

Let $I=$ moment of inertia of the pendulum

$\tau=$ modulus of torsion of the filament (moment required to give Then: the beam an angular displacement of 1 radian)

$$
\begin{gathered}
\text { Kinetic energy of pendulum }=\frac{I}{2}\left(\frac{d \theta}{d t}\right)^{2} \\
\text { Potential energy due to filament }=\frac{1}{2} \tau \theta^{2},
\end{gathered}
$$

and it remains to find an expression for the potential energy of attraction as a function of $\theta$. 
To find the potential energy of attraction we must take the sum of the attractions of the cylinders for the whole moving system, beam and balls. The attraction for the balls is comparatively simple, as we may suppose the mass of either ball concentrated at its center. For the beam the calculation is more laborious, as it is necessary to imagine the beam divided into a number of elementary segments so small that it is permissible to assume the attractive force concentrated at the center of a segment. We shall consider first the attraction of one cylinder on the pendulum.

Let $V_{0}$ be the potential of the cylinder at the center of any element of the pendulum (ball or beam segment) when the beam is in an equilibrium position (near or far) where $\theta=0$, and let $V_{\theta}$ be the potential when the beam is displaced from equilibrium by an angle $\theta$. Then, by Taylor's theorem:

$$
V_{\theta}-V_{0}=\theta \frac{d V_{0}}{d \theta}+\frac{\theta^{2}}{2} \frac{d^{2} V_{0}}{d \theta^{2}}
$$

neglecting terms in $\theta^{3}$ and higher powers. This is a valid procedure, not because the pendulum actually swings through a small angle, but because its time of swing is reduced empirically to its value $T_{0}$ for an infinitesimal arc before being used in the calculations.

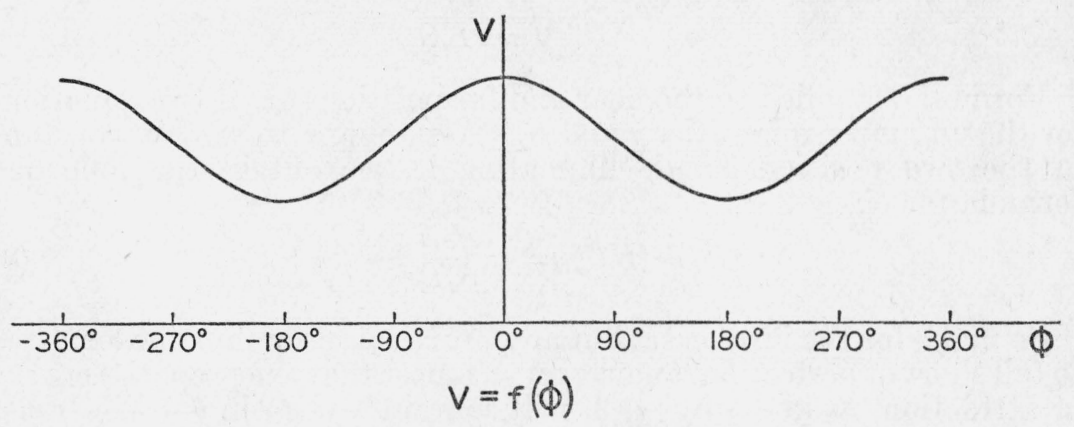

Figure 5.-Curve of potential.

If $m=$ mass of an element of the pendulum, $-m\left(V_{\theta}-V_{0}\right)$ will be the potential energy of attraction of the cylinder for this element. Taking into account both cylinders and all elements of the pendulum:

$$
\text { Potential energy of attraction }=-\Sigma\left(m \theta \frac{d V_{0}}{d \theta}+\frac{m}{2} \theta^{2} \frac{d^{2} V_{0}}{d \theta^{2}}\right) .
$$

In the ideal case of perfect centering of the pendulum, and symmetry and parallelism of the cylinders, the terms in $d V_{0} / d \theta$ disappear in both near and far positions. In the near position, $V_{0}$ is a maximum for the position angle $\phi=0$, and $d V_{0} / d \phi=0$; and for the position angle $\phi=180^{\circ}, V_{0}$ will be a minimum and $d V_{0} / d \phi$ will again be zero. And since, as we have seen (fig. 4 ), $d \phi=d \theta$, all terms in $d V_{0} / d \theta$ are individually zero in the near position.

In the far position (ideal case), for the equilibrium angles $\phi=90^{\circ}$ and $\phi=270^{\circ}, d V_{0} / d \phi$ will not be zero, but will have equal and opposite values in these two positions (fig. 5). In consequence, the terms in $d V / d \theta$ will mutually cancel out in the summation. 
In the ideal case, therefore, we have in both near and far positions:

$$
\text { Potential energy of attraction }=-\sum \frac{m}{2}\left(\theta^{2} \frac{d^{2} V_{0}}{d \theta^{2}}\right)=-\frac{1}{2} K G \theta^{2} \text {, }
$$

where $G$ is the constant of gravitation and $K$ is a calculable function of the masses and dimensions in the apparatus.

Equation 1 now becomes, in the ideal case:

$$
\frac{I}{2}\left(\frac{d \theta}{d t}\right)^{2}+\frac{1}{2} \tau \theta^{2}-\frac{1}{2} K G \theta^{2}=\text { constant. }
$$

Differentiating and reducing:

whose solution is:

$$
\frac{d^{2} \theta}{d t^{2}}+\frac{\tau-K G}{I} \theta=0
$$

with a periodic time:

$$
\theta=A \sin \left(\sqrt{\frac{\tau-K G}{I}}\right) t
$$

$$
T=2 \pi \sqrt{\frac{I}{\tau-K G}} \cdot
$$

Formula 7 applied to the near and far positions gives two equations for the unknown quantities $\tau$ and G. Assuming $\tau$ to remain constant in the two positions, and eliminating it, we obtain the following formula for $G$ :

$$
(K G)_{F}-(K G)_{N}=4 \pi^{2}\left(\frac{I}{T_{N}^{2}}-\frac{I}{T_{F}^{2}}\right)
$$

So much for the ideal case; but any practical setup must be expected to fall short of perfection, and we must expect that the potential energy of attraction, as given by eq 3 , will contain a term in $\theta$. We must therefore rewrite eq 4 as follows:

$$
\text { Potential energy of attraction }=-\frac{1}{2} K G \theta^{2}+Q \theta,
$$

in which $Q$, like $K$, is a calculable function of the masses and dimensions in the apparatus. Equation 1 now becomes, in the practical case:|

$$
\frac{I}{2}\left(\frac{d \theta}{d t}\right)^{2}+\frac{1}{2}(\tau-K G) \theta^{2}+Q \theta=\text { constant. }
$$

Differentiating and reducing:

$$
\frac{d^{2} \theta}{d t^{2}}+\frac{\tau-K G}{I} \theta+\frac{Q}{I}=0
$$

whose solution is:

$$
\theta=A \sin \left(\sqrt{\frac{\tau-K G}{I}}\right) t-\frac{Q}{\tau-K G}
$$


which has the same periodic time as in the ideal case (formula 6). It appears, then, that even in cases where a term in $\theta$ is present, we may follow the same procedure for the calculation of $G$ as when it is absent.

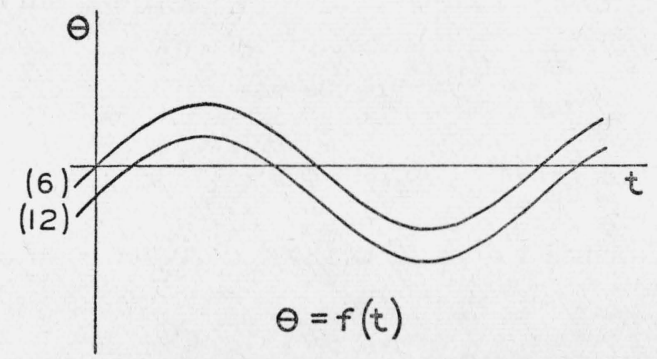

Figure 6.-Ideal and practical time-of-swing curves.

The relation between the ideal and the practical solutions is shown in figure 6 . The ideal solution (6) is a sine curve which is symmetrical with respect to the axis of $t$; the practical solution (12) is a similar sine curve at a constant distance below the other. The curve has the same periodic time, but while the "half-periods" in the practical case are alternately long and short, in the ideal case they are equal.

In the present work the $\theta$ term is insignificant compared with the $\theta^{2}$ term. A calculation made for one of the near positions showed that the ratio of the coefficients of the two terms was about 1 to 40,000 .

In order to apply formula 8 to the calculation of $G$, it is necessary to compute the value of

$$
K G=\sum\left(m \frac{d^{2} V_{0}}{d \theta^{2}}\right)
$$

for both the near and the far positions. The 1930 paper gives a formula for $V$ at any point exterior to a cylinder (eq 9, p. 1264-66), and by differentiation with respect to $\phi$, we can obtain from this the value of

at any desired point.

$$
\frac{d^{2} V}{d \phi^{2}}\left(\equiv \frac{d^{2} V}{d \theta^{2}}\right)
$$

Using the notation given in figure 3 for the fundamental quantities, we obtain the following relations:

$$
\left.\begin{array}{rl}
C_{\phi} & =C_{0}+R(1-\cos \phi) \\
\frac{d C_{\phi}}{d \phi} & =R \sin \phi \\
\frac{d^{2} C_{\phi}}{d \phi^{2}} & =R \cos \phi
\end{array}\right\}
$$

At the four equilibrium values of $\phi$ these derivatives of $C$ have the following values:

$\left.\begin{array}{rrr}\phi & \frac{d C_{\phi}}{d \phi} & \frac{d^{2} C_{\phi}}{d \phi^{2}} \\ \hline 0^{\circ} & 0 & R \\ 90^{\circ} & R & 0 \\ 180^{\circ} & 0 & -R \\ 270^{\circ} & -R & 0\end{array}\right\}$


We have also the following values of $l_{1}$ and $l_{2}$, and their derivatives as functions of $\phi$, in which $\Delta_{1}$ and $\Delta_{2}$ denote errors in centering:

$$
\left.\begin{array}{c}
l_{1}=\frac{L}{2} \pm \Delta_{1}-R \sin \phi \quad l_{2}=-\left(\frac{L}{2} \pm \Delta_{2}+R \sin \phi\right) \\
\frac{d l_{1}}{d \phi}=\frac{d l_{2}}{d \phi}=-R \cos \phi \\
\frac{d^{2} l_{1}}{d \phi^{2}}=\frac{d^{2} l_{2}}{d \phi^{2}}=R \sin \phi
\end{array}\right\}
$$

At the equilibrium values of $\phi$ these derivatives of $\phi$ have the following values:

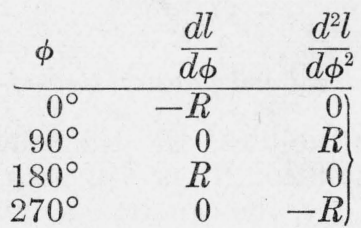

And since, for both subscripts, $r^{2}=C_{\phi}{ }^{2}+l^{2}$, we obtain

$$
\begin{aligned}
& \frac{d r}{d \phi}=\frac{C_{\phi}}{r} \frac{d C_{\phi}}{d \phi}+\frac{l}{r} \frac{d l}{d \phi} \\
& \frac{d^{2} r}{d \phi^{2}}=\left\{\begin{array}{l}
+\frac{C_{\phi}}{r} \frac{d^{2} C_{\phi}}{d \phi^{2}}+\frac{1}{r}\left(\frac{d C_{\phi}}{d \phi}\right)^{2}-\frac{C_{\phi}}{r^{2}} \frac{d r}{d \phi} \frac{d C_{\phi}}{d \phi} \\
+\frac{l}{r} \frac{d^{2} l}{d \phi^{2}}+\frac{1}{r}\left(\frac{d l}{d \phi}\right)^{2}-\frac{l}{r^{2}} \frac{d r}{d \phi} \frac{d l}{d \phi}
\end{array}\right\}
\end{aligned}
$$

At the equilibrium values of $\phi$ these derivatives of $r$ have the following values:

\begin{tabular}{c|c|c}
\hline$\phi$ & $\frac{d r}{d \phi}$ & $\frac{d^{2} r}{d \phi^{2}}$ \\
\hline $0^{\circ}$ & $-R \frac{l}{r}$ & $R \frac{C_{\phi}}{r}+\frac{R^{2}}{r}-R^{2} \frac{l^{2}}{r^{3}}$ \\
\hline $180^{\circ}$ & $\frac{R^{2}}{r}-R^{2} \frac{C_{\phi}^{2}}{r^{3}}+R \frac{l}{r}$ \\
$270^{\circ}$ & $-R \frac{C_{\phi}}{r}$ & $-R \frac{C_{\phi}}{r}+\frac{R^{2}}{r}-R^{2} \frac{l^{2}}{r^{3}}$ \\
$-R \frac{C_{\phi}}{r}$ & $\frac{R^{2}}{r}-\frac{R^{2} C_{\phi}^{2}}{r^{3}}-R \frac{l}{r}$
\end{tabular} \mid

The foregoing formulas will enable us to obtain $d^{2} V / d \phi^{2}$ from the formula for $V$ in the 1930 paper. In performing this differentiation it is to be remembered that a change in the sign of $l_{2}$ has been made 
for the present work. We thus obtain the following formula (19) for $d^{2} V / d \phi^{2}$. For convenience in printing, only the first term is given in full. In this it will be seen that to each term in $l_{2}$ and $r_{2}$ there corresponds a similar term in $l_{1}$ and $r_{1}$, but with opposite sign. The same holds true in all terms after the first, and in these terms, for brevity, the terms in $l_{1}$ and $r_{1}$ are omitted.

It will be convenient to classify all terms after the first according to the different derivatives which they contain. These are five in number:

$$
\frac{d^{2} l}{d \phi^{2}}, \frac{d^{2} r}{d \phi^{2}},\left(\frac{d r}{d \phi}\right)^{2},\left(\frac{d l}{d \phi}\right)^{2} \text { and } \frac{d r}{d \phi} \frac{d l}{d \phi}
$$

And finally, all terms, including the first, are to be multiplied by $2 \pi \rho a^{2} G$, where $\rho$ is the density of the cylinder. (This factor is incorrectly given with a negative sign in the 1930 paper.)

$\frac{1}{2 \pi \rho a^{2} G} \frac{d^{2} V}{d \phi^{2}}$

FIRST TERM

$\frac{d C_{\phi}}{d \phi}\left[-\frac{1}{C_{\phi}{ }^{3}}\left(l_{2} \frac{d r_{2}}{d \phi}-l_{1} \frac{d r_{1}}{d \phi}-r_{2} \frac{d l_{2}}{d \phi}+r_{1} \frac{d l_{1}}{d \phi}\right)\right]+\frac{d^{2} r_{2}}{d \phi^{2}} \frac{l_{2}}{2 C_{\phi}^{2}}-\frac{d^{2} r_{1}}{d \phi^{2}} \frac{l_{1}}{2 C_{\phi}^{2}}-$

Term

$$
\frac{d^{2} l_{2}}{d \phi^{2}} \frac{r_{2}}{2 C_{\phi}^{2}}+\frac{d^{2} l_{1}}{d \phi^{2}} \frac{r_{1}}{2 C_{\phi}^{2}} \cdot
$$

TERMS IN $\frac{d^{2} l}{d \phi^{2}}$

2. $\quad \frac{a^{2}}{16} \frac{d^{2} l_{2}}{d \phi^{2}}\left(-\frac{1}{r_{2}^{3}}\right)$

3. $\quad \frac{a^{4}}{128} \frac{d^{2} l_{2}}{d \phi^{5}}\left(+\frac{15 l_{2}^{2}}{r_{2}{ }^{7}}-\frac{3}{r_{2}^{5}}\right)$

4. $\quad \frac{5 a^{6}}{2048} \frac{d^{2} l_{2}}{d \phi^{2}}\left(-\frac{105 l_{2}^{4}}{r_{2}{ }^{11}}+\frac{70 l_{2}{ }^{2}}{r_{2}{ }^{9}}-\frac{5}{r_{2}{ }^{7}}\right)$

5. $\quad \frac{7 a^{8}}{32768} \frac{d^{2} l_{2}}{d \phi^{2}}\left(+\frac{3003 l_{2}{ }^{6}}{r_{2}{ }^{15}}-\frac{3465 l_{2}^{4}}{r_{2}{ }^{13}}+\frac{945 l_{2}{ }^{2}}{r_{2}{ }^{11}}-\frac{35}{r_{2}{ }^{9}}\right)$

6. $\quad \frac{21 a^{10}}{262144} \frac{d^{2} l_{2}}{d \phi^{2}}\left(-\frac{21879 l_{2}{ }^{8}}{r_{2}{ }^{19}}+\frac{36036 l_{2}{ }^{6}}{r_{2}{ }^{17}}-\frac{18018 l_{2}{ }^{4}}{r_{2}{ }^{15}}+\frac{2772 l_{2}{ }^{2}}{r_{2}{ }^{13}}-\frac{63}{r_{2}{ }^{11}}\right)$

7. $\frac{33 a^{12}}{2097152} \frac{d^{2} l_{2}}{d \phi^{2}}\left(+\frac{323323 l_{2}{ }^{10}}{r_{2}{ }^{23}}-\frac{692835 l_{2}{ }^{8}}{r_{2}{ }^{21}}+\frac{510510 l_{2}{ }^{6}}{r_{2}{ }^{19}}\right.$

$$
\left.-\frac{150150 l_{2}^{4}}{r_{2}{ }^{17}}+\frac{15015 l_{2}^{2}}{r_{2}^{15}}-\frac{231}{r_{2}^{13}}\right)
$$

8. $\frac{429 a^{14}}{67108864} \frac{d^{2} l_{2}}{d \phi^{2}}\left(-\frac{2414425 l_{2}^{12}}{r_{2}{ }^{27}}+\frac{6374082 l_{2}^{10}}{r_{2}^{25}}-\frac{6235515 l_{2}{ }^{8}}{r_{2}^{23}}\right.$

$$
\left.+\frac{2771340 l_{2}{ }^{6}}{r_{2}{ }^{21}}-\frac{546975 l_{2}^{4}}{r_{2}{ }^{19}}+\frac{38610 l_{2}^{2}}{r_{2}{ }^{17}}-\frac{429}{r_{2}{ }^{15}}\right)
$$


9. $\quad \frac{715 a^{16}}{2147483648} \frac{d^{2} l_{2}}{d \phi^{2}}\left(+\frac{145422675 l_{2}^{14}}{r_{2}^{31}}-\frac{456326325 l_{2}^{12}}{r_{2}^{29}}+\frac{557732175 l_{2}{ }^{10}}{r_{2}^{27}}\right.$

$$
\left.-\frac{334639305 l_{2}^{8}}{r_{2}{ }^{25}}+\frac{101846745 l_{2}^{6}}{r_{2}{ }^{23}}-\frac{14549535 l_{2}^{4}}{r_{2}{ }^{21}}+\frac{765765 l_{2}{ }^{2}}{r_{2}{ }^{19}}-\frac{6435}{r_{2}{ }^{17}}\right)
$$

10. $\frac{2431 a^{18}}{17179869184} \frac{d^{2} l_{2}}{d \phi^{2}}\left(-\frac{1101980715 l_{2}^{16}}{r_{2}^{35}}+\frac{4007202600 l_{2}^{14}}{r_{2}^{33}}\right.$

$$
\begin{aligned}
& -\frac{5881539300 l_{2}^{12}}{r_{2}^{31}}+\frac{4461857400 l_{2}^{10}}{r_{2}{ }^{29}}-\frac{1859107250 l_{2}{ }^{8}}{r_{2}{ }^{27}} \\
& \left.\quad+\frac{416440024 l_{2}^{6}}{r_{2}{ }^{25}}-\frac{45265220 l_{2}^{4}}{r_{2}{ }^{23}}+\frac{1847560 l_{2}{ }^{2}}{r_{2}{ }^{21}}-\frac{12155}{r_{2}{ }^{19}}\right)
\end{aligned}
$$

2. 0.0625

3. $\quad 0.1171875 \quad 0.0234375$

4. $\quad 0.2563477 \quad 0.1708984$

5. $\quad 0.6415100$

0.7402039

0.0122070

6. 1.752697

2. 886795

0.2018738

0.0074768

7. 5.087690

10. 90219

1. 443398

0.2220612

8. 033195

2. 362704

0. 0050468

8. 15.43445

40. 74694

39. 86114

17. 71606

0. 2362704

9. 48.41816

151. 9329

185. 6957

0. 2468182

0. 0036349

10. 155.9334

567. 0305

48. 44236

58. 92744

832. 2544

111. 4174

25. 49598

631. 3654

3. 496591

0. 0027424

33. 90965

0. 2142519

64. 05156

2. 614350

263. 0689

0. 0171997

Term TERMS IN $\frac{d^{2} r}{d \phi^{2}}$

2. $\quad \frac{a^{2}}{16} \frac{d^{2} r_{2}}{d \phi^{2}}\left(+\frac{3 l_{2}}{r_{2}{ }^{4}}\right)$.

3. $\quad \frac{a^{4}}{128} \frac{d^{2} r_{2}}{d \phi^{2}}\left(-\frac{35 l_{2}^{3}}{r_{2}^{8}}+\frac{15 l_{2}}{r_{2}^{6}}\right)$.

4. $\quad \frac{5 a^{6}}{2048} \frac{d^{2} r_{2}}{d \phi^{2}}\left(+\frac{231 l_{2}^{5}}{r_{2}{ }^{2}}-\frac{210 l_{2}^{3}}{r_{2}{ }^{10}}+\frac{35 l_{2}}{r_{2}{ }^{8}}\right)$.

5. $\quad \frac{7 a^{8}}{32768} \frac{d^{2} r_{2}}{d \phi^{2}}\left(-\frac{6435 l_{2}^{7}}{r_{2}{ }^{16}}+\frac{9009 l_{2}{ }^{5}}{r_{2}{ }^{14}}-\frac{3465 l_{2}{ }^{3}}{r_{2}{ }^{12}}+\frac{315 l_{2}}{r_{2}{ }^{10}}\right)$.

6. $\frac{21 a^{10}}{262144} \frac{d^{2} r_{2}}{d \phi^{2}}\left(+\frac{46189 l_{2}{ }^{9}}{r_{2}{ }^{20}}-\frac{87516 l_{2}{ }^{7}}{r_{2}{ }^{18}}+\frac{54054 l_{2}{ }^{5}}{r_{2}{ }^{16}}-\frac{12012 l_{2}{ }^{3}}{r_{2}{ }^{14}}+\frac{693 l_{2}}{r_{2}{ }^{12}}\right)$.

7. $\quad \frac{33 a^{12}}{2097152} \frac{d^{2} r_{2}}{d \phi^{2}}\left(-\frac{676039 l_{2}^{11}}{r_{2}{ }^{24}}+\frac{1616615 l_{2}^{9}}{r_{2}^{22}}-\frac{1385670 l_{2}^{7}}{r_{2}^{20}}\right.$

$$
\left.+\frac{510510 l_{2}^{5}}{r_{2}{ }^{18}}-\frac{75075 l_{2}^{3}}{r_{2}^{16}}+\frac{3003 l_{2}}{r_{2}^{14}}\right)
$$


8. $\quad \frac{429 a^{14}}{67108864} \frac{d^{2} r_{2}}{d \phi^{2}}\left(+\frac{5014575 l_{2}^{13}}{r_{2}{ }^{28}}-\frac{14486550 l_{2}^{11}}{r_{2}{ }^{26}}+\frac{15935205 l_{2}{ }^{9}}{r_{2}{ }^{24}}\right.$

$$
\left.-\frac{8314020 l_{2}^{7}}{r_{2}^{22}}+\frac{2078505 l_{2}^{5}}{r_{2}{ }^{20}}-\frac{218790 l_{2}^{3}}{r_{2}{ }^{18}}+\frac{6435 l_{2}}{r_{2}{ }^{16}}\right)
$$

Term

9. $\quad \frac{715 a^{16}}{2147483648} \frac{d^{2} r_{2}}{d \phi^{2}}\left(-\frac{300540195 l_{2}^{15}}{r_{2}{ }^{32}}+\frac{1017958725 l_{2}^{13}}{r_{2}{ }^{30}}-\frac{1368978975 l_{2}^{11}}{r_{2}^{28}}\right.$ $\left.+\frac{929553625 l_{2}^{9}}{r_{2}^{26}}-\frac{334639305 l_{2}^{7}}{r_{2}^{24}}+\frac{61108047 l_{2}^{5}}{r_{2}{ }^{22}}-\frac{4849845 l_{2}^{3}}{r_{2}{ }^{20}}+\frac{109395 l_{2}}{r_{2}{ }^{18}}\right)$

10. $\frac{2431 a^{18}}{17179869184} \frac{d^{2} r_{2}}{d \phi^{2}}\left(+\frac{2268783825 l_{2}^{17}}{r_{2}^{36}}-\frac{8815845720 l_{2}^{15}}{r_{2}^{34}}\right.$

$$
\begin{aligned}
& +\frac{14025209100 l_{2}^{13}}{r_{2}^{32}}-\frac{11763078600 l_{2}^{11}}{r_{2}^{30}}+\frac{5577321750 l_{2}^{9}}{r_{2}^{28}} \\
& \left.-\frac{1487285800 l_{2}^{7}}{r_{2}{ }^{26}}+\frac{208220012 l_{2}^{5}}{r_{2}{ }^{24}}-\frac{12932920 l_{2}{ }^{3}}{r_{2}{ }^{22}}+\frac{230945 l_{2}}{r_{2}{ }^{20}}\right)
\end{aligned}
$$

2. 0.1875

3. $\quad 0.2734375$

NUMERICAL FACTORS

4. 0.5639648

0.1171875

5. $\quad 1.374664$

0.5126953

6. 3.700138

1.924530

0.0854492

7.010788

0.7402039

0.0672913

7. 10.63790

25.43845

21.80439

0.9622650

8.033195

0.0555153

8. 32.05616

92.60669

101.8674

9. 100.0642

338.9271

455.7986

53.14819

1.398637

309.4929

20.34579

10. 321.0393

29.46372

1.614745

1664.509

1.830045

1.181352

0.0472541

13.28705

0.0411364

111.4174

0.0364228

789.2068

0.0326794

Term $\operatorname{TERMS~IN~}\left(\frac{d r}{d \phi}\right)^{2}$

2. $\frac{a^{2}}{16}\left(\frac{d r_{2}}{d \phi}\right)^{2}\left(-\frac{12 l_{2}}{r_{2}^{5}}\right)$.

3. $\frac{a^{4}}{128}\left(\frac{d r_{2}}{d \phi}\right)^{2}\left(+\frac{280 l_{2}{ }^{3}}{r_{2}{ }^{9}}-\frac{90 l_{2}}{r_{2}{ }^{7}}\right)$.

4. $\quad \frac{5 a^{6}}{2048}\left(\frac{d r_{2}}{d \phi}\right)^{2}\left(-\frac{2772 l_{2}^{5}}{r_{2}{ }^{13}}+\frac{2100 l_{2}^{3}}{r_{2}{ }^{11}}-\frac{280 l_{2}}{r_{2}{ }^{9}}\right)$.

5. $\frac{7 a^{8}}{32768}\left(\frac{d r_{2}}{d \phi}\right)^{2}\left(+\frac{102960 l_{2}^{7}}{r_{2}^{17}}-\frac{126126 l_{2}^{5}}{r_{2}^{15}}+\frac{41580 l_{2}^{3}}{r_{2}{ }^{13}}-\frac{3150 l_{2}}{r_{2}^{11}}\right)$

$463607-42-2$ 
16 Journal of Research of the National Bureau of Standards

6. $\quad \frac{21 a^{10}}{262144}\left(\frac{d r_{2}}{d \phi}\right)^{2}\left(-\frac{923780 l_{2}{ }^{9}}{r_{2}{ }^{21}}+\frac{1575288 l_{2}{ }^{7}}{r_{2}{ }^{19}}-\frac{864864 l_{2}{ }^{5}}{r_{2}{ }^{17}}\right.$

$$
\left.+\frac{168168 l_{2}^{3}}{r_{2}{ }^{15}}-\frac{8316 l_{2}}{r_{2}{ }^{13}}\right)
$$

7. $\frac{33 a^{12}}{2097152}\left(\frac{d r_{2}}{d \phi}\right)^{2}\left(+\frac{16224936 l_{2}^{11}}{r_{2}^{25}}-\frac{35565530 l_{2}{ }^{2}}{r_{2}^{23}}+\frac{27713400 l_{2}^{7}}{r_{2}^{21}}\right.$

$$
\left.-\frac{9189180 l_{2}^{5}}{r_{2}{ }^{19}}+\frac{1201200 l_{2}^{3}}{r_{2}{ }^{17}}-\frac{42042 l_{2}}{r_{2}^{15}}\right)
$$

8. $\frac{429 a^{14}}{67108864}\left(\frac{d r_{2}}{d \phi}\right)^{2}\left(-\frac{140408100 l_{2}^{13}}{r_{2}^{29}}+\frac{376650300 l_{2}^{11}}{r_{2}^{27}}-\frac{382444920 l_{2}^{9}}{r_{2}^{25}}\right.$

$$
\left.+\frac{182908440 l_{2}^{7}}{r_{2}{ }^{23}}-\frac{41570100 l_{2}^{5}}{r_{2}{ }^{21}}+\frac{3938220 l_{2}{ }^{3}}{r_{2}{ }^{19}}-\frac{102960 l_{2}}{r_{2}{ }^{17}}\right) \text {. }
$$

9. $\quad \frac{715 a^{16}}{2147483648}\left(\frac{d r_{2}}{d \phi}\right)^{2}\left(+\frac{9617286240 l_{2}^{15}}{r_{2}^{33}}-\frac{30538761750 l_{2}^{13}}{r_{2}{ }^{31}}\right.$

$$
\begin{aligned}
+\frac{38331411300 l_{2}{ }^{11}}{r_{2}{ }^{29}} & -\frac{24168394250 l_{2}{ }^{9}}{r_{2}{ }^{27}}+\frac{8031343320 l_{2}{ }^{7}}{r_{2}{ }^{25}} \\
& \left.-\frac{1344377034 l_{2}{ }^{5}}{r_{2}{ }^{23}}+\frac{96996900 l_{2}{ }^{3}}{r_{2}{ }^{21}}-\frac{1969110 l_{2}}{r_{2}{ }^{19}}\right) .
\end{aligned}
$$

10. $\frac{2431 a^{18}}{17179869184}\left(\frac{d r_{2}}{d \phi}\right)^{2}\left(-\frac{81676217700 l_{2}^{17}}{r_{2}{ }^{17}}+\frac{299738754480 l_{2}^{15}}{r_{2}{ }^{35}}\right.$

$$
\begin{gathered}
-\frac{448806691200 l_{2}^{13}}{r_{2}^{33}}+\frac{352892358000 l_{2}{ }^{11}}{r_{2}^{31}}-\frac{156165009000 l_{2}{ }^{9}}{r_{2}{ }^{29}} \\
\left.+\frac{38669430800 l_{2}^{7}}{r_{2}{ }^{27}}-\frac{4997280288 l_{2}^{5}}{r_{2}{ }^{25}}+\frac{284524240 l_{2}^{3}}{r_{2}{ }^{23}}-\frac{4618900 l_{2}}{r_{2}^{21}}\right)
\end{gathered}
$$

$2 . \quad 0.75$

$\begin{array}{lll}3 . & 2.1875 & 0.703125\end{array}$

$\begin{array}{lll}4 . & 6.767578 & 5.126953\end{array}$

5. $\quad 21.99463 \quad 26.94342$

6. $\quad 74.00276 \quad 126.1942$

NUMERICAL FACTORS

7. $255.3095 \quad 559.6459$

0.6835938

8. 882446

0. 6729126

69. $28308 \quad 13.47171$

0. 6661835

436.0877 144. 5975

18. 90163

8.

$\begin{array}{lll}\text { 8. } & 897.5726 & 2407.77\end{array}$

2444. 817 1169. 260

0. 6615572

9. $3202.054 \quad 10167.81$

25. 17546

265. 7409

$\begin{array}{llll} & 12762.36 & 8046.814 & 2674.018\end{array}$

10. $11557.42 \quad 42413.88 \quad 63507.41 \quad 49935.27 \quad 22097.79$

$\begin{array}{lll}447.6074 & 32.29491 & 0.6556109\end{array}$ $\begin{array}{lll}5471.834 & 707.1293 & 40.26098\end{array}$

0. 6535874

Term

2. 0 Terms IN $\left(\frac{d l}{d \phi}\right)^{2}$

3. $\frac{a^{4}}{128}\left(\frac{d l_{2}}{d \phi}\right)^{2}\left(+\frac{30 l_{2}}{r_{2}^{7}}\right)$ 
4. $\quad \frac{5 a^{6}}{2048}\left(\frac{d l_{2}}{d \phi}\right)^{2}\left(-\frac{420 l_{2}^{3}}{r_{2}{ }^{11}}+\frac{140 l_{2}}{r_{2}{ }^{9}}\right)$

5. $\quad \frac{7 a^{8}}{32768}\left(\frac{d l_{2}}{d \phi}\right)^{2}\left(+\frac{18018 l_{2}{ }^{5}}{r_{2}{ }^{15}}-\frac{13860 l_{2}^{3}}{r_{2}{ }^{13}}+\frac{1890 l_{2}}{r_{2}{ }^{11}}\right)$

6. $\frac{21 a^{10}}{262144}\left(\frac{d l_{2}}{d \phi}\right)^{2}\left(-\frac{175032 l_{2}{ }^{7}}{r_{2}{ }^{19}}+\frac{216216 l_{2}^{5}}{r_{2}{ }^{17}}-\frac{72072 l_{2}^{3}}{r_{2}{ }^{15}}+\frac{5544 l_{2}}{r_{2}{ }^{13}}\right)$

7. $\frac{33 a^{12}}{2097152}\left(\frac{d l_{2}}{d \phi}\right)^{2}\left(+\frac{3233230 l_{2}{ }^{9}}{r_{2}^{23}}-\frac{5542680 l_{2}{ }^{7}}{r_{2}^{21}}\right.$

$$
\left.+\frac{3063060 l_{2}^{5}}{r_{2}{ }^{19}}-\frac{600600 l_{2}^{3}}{r_{2}{ }^{17}}+\frac{30030 l_{2}}{r_{2}{ }^{15}}\right)
$$

8. $\frac{429 a^{14}}{67108864}\left(\frac{d l_{2}}{d \phi}\right)^{2}\left(-\frac{28973100 l_{2}^{11}}{r_{2}{ }^{27}}+\frac{63740820 l_{2}^{9}}{r_{2}{ }^{25}}-\frac{49884120 l_{2}{ }^{11}}{r_{2}{ }^{23}}\right.$

$$
\left.+\frac{16628040 l_{2}^{5}}{r_{2}{ }^{21}}-\frac{2187900 l_{2}^{3}}{r_{2}{ }^{19}}+\frac{77220 l_{2}}{r_{2}{ }^{17}}\right)
$$

9. $\frac{715 a^{16}}{2147483648}\left(\frac{d l_{2}}{d \phi}\right)^{2}\left(+\frac{2035917450 l_{2}^{13}}{r_{2}{ }^{31}}-\frac{5475915900 l_{2}^{11}}{r_{2}^{29}}\right.$

$$
\begin{aligned}
& +\frac{5577321750 l_{2}^{9}}{r_{2}{ }^{27}}-\frac{2677114440 l_{2}{ }^{7}}{r_{2}{ }^{25}}+\frac{611080470 l_{2}{ }^{5}}{r_{2}{ }^{23}} \\
& \left.-\frac{58198140 l_{2}{ }^{3}}{r_{2}{ }^{21}}+\frac{1531530 l_{2}}{r_{2}{ }^{19}}\right)
\end{aligned}
$$

10. $\frac{2431 a^{18}}{17179869184}\left(\frac{d l_{2}}{d \phi}\right)^{2}\left(-\frac{17631691440 l_{2}^{15}}{r_{2}^{35}}+\frac{56100836400 l_{2}^{13}}{r_{2}^{33}}\right.$

$-\frac{70578471600 l_{2}{ }^{11}}{r_{2}{ }^{31}}+\frac{44618574000 l_{2}{ }^{9}}{r_{2}{ }^{29}}-\frac{14872858000 l_{2}^{7}}{r_{2}{ }^{27}}$
$\left.+\frac{2498640144 l_{2}{ }^{5}}{r_{2}{ }^{25}}-\frac{181060880 l_{2}^{3}}{r_{2}{ }^{23}}+\frac{3695120 l_{2}}{r_{2}{ }^{21}}\right)$

2. 0 .

3. $\quad 0.234375$

4. $\quad 1.025391 \quad 0.3417969$

$\begin{array}{llll}\text { 5. } & 3.849060 & 2.960815 & 0.4037476\end{array}$

6. $14.02158 \quad 17.32077 \quad 5.773590$

$\begin{array}{llll}7 . & 50.87690 & 87.21754 & 48.19917\end{array}$

8. $185.2134 \quad 407.4694 \quad 318.8891$

$\begin{array}{cc}0.4441223 & \\ 9.450817 & 0.4725409 \\ 106.2964 & 13.98637 \\ & 0.4936364 \\ 891.3394 & 203.4579 \\ 19.37694 & 0.5099196 \\ 6313.654 & 2104.551\end{array}$

10. $2494.934 \quad 7938.427 \quad 9987.053$

353.5646

25.62063

0.5228699 
18 Journal of Research of the National Bureau of Standards

Term $\operatorname{TERMS~IN~}\left(\frac{d r}{d \phi} \frac{d l}{d \phi}\right)$

2. $\quad \frac{a^{2}}{16}\left(\frac{d r_{2}}{d \phi} \frac{d l_{2}}{d \phi}\right)\left(+\frac{6}{r_{2}^{4}}\right)$

3. $\frac{a^{4}}{128}\left(\frac{d r_{2}}{d \phi} \frac{d l_{2}}{d \phi}\right)\left(-\frac{210 l_{2}^{2}}{r_{2}^{8}}+\frac{30}{r_{2}{ }^{6}}\right)$

4. $\quad \frac{5 a^{6}}{2048}\left(\frac{d r_{2}}{d \phi} \frac{d l_{2}}{d \phi}\right)\left(+\frac{2310 l_{2}^{4}}{r_{2}{ }^{12}}-\frac{1260 l_{2}{ }^{2}}{r_{2}{ }^{10}}+\frac{70}{r_{2}{ }^{8}}\right)$

5. $\quad \frac{7_{a}{ }^{8}}{32768}\left(\frac{d r_{2}}{d \phi} \frac{d l_{2}}{d \phi}\right)\left(-\frac{90090 l_{2}{ }^{6}}{r_{2}{ }^{16}}+\frac{90090 l_{2}{ }^{4}}{r_{2}{ }^{14}}-\frac{20790 l_{2}{ }^{2}}{r_{2}{ }^{12}}+\frac{630}{r_{2}{ }^{10}}\right)$

6. $\frac{21 a^{10}}{262144}\left(\frac{d r_{2}}{d \phi} \frac{d l_{2}}{d \phi}\right)\left(+\frac{831402 l_{2}^{8}}{r_{2}{ }^{20}}-\frac{1225224 l_{2}^{6}}{r_{2}^{18}}+\frac{540540 l_{2}^{4}}{r_{2}{ }^{16}}\right.$ $\left.-\frac{72072 l_{2}^{2}}{r_{2}{ }^{14}}+\frac{1386}{r_{2}^{12}}\right)$

7. $\frac{33 a^{12}}{2097152}\left(\frac{d r_{2}}{d \phi} \frac{d l_{2}}{d \phi}\right)\left(-\frac{14872858 l_{2}{ }^{10}}{r_{2}{ }^{24}}+\frac{29099070 l_{2}{ }^{8}}{r_{2}{ }^{22}}-\frac{19399380 l_{2}{ }^{6}}{r_{2}{ }^{20}}\right.$ $\left.+\frac{5105100 l_{2}^{4}}{r_{2}{ }^{18}}-\frac{450450 l_{2}^{2}}{r_{2}{ }^{16}}+\frac{6006}{r_{2}{ }^{14}}\right)$

8. $\quad \frac{429 a^{14}}{67108864}\left(\frac{d r_{2} d l_{2}}{d \phi d \phi}\right)\left(+\frac{130378950 l_{2}^{12}}{r_{2}{ }^{28}}-\frac{318704100 l_{2}^{10}}{r_{2}^{26}}+\frac{286833690 l_{2}^{8}}{r_{2}{ }^{24}}\right.$ $\left.-\frac{116396280 l_{2}^{6}}{r_{2}^{22}}+\frac{20785050 l_{2}^{4}}{r_{2}{ }^{20}}-\frac{1312740 l_{2}^{2}}{r_{2}{ }^{18}}+\frac{12870}{r_{2}^{16}}\right)$

9. $\quad \frac{715 a^{16}}{2147483648}\left(\frac{d r_{2} d l_{2}}{d \phi d \phi}\right)\left(-\frac{9016205850 l_{2}^{14}}{r_{2}^{32}}+\frac{26466926850 l_{2}^{12}}{r_{2}^{30}}\right.$ $-\frac{30117537450 l_{2}^{10}}{r_{2}{ }^{28}}+\frac{16731965250 l_{2}^{8}}{r_{2}^{26}}-\frac{4684950270 l_{2}^{6}}{r_{2}^{24}}$ $\left.+\frac{611080470 l_{2}^{4}}{r_{2}^{22}}-\frac{29099070 l_{2}^{2}}{r_{2}^{20}}+\frac{218790}{r_{2}^{18}}\right)$

10. $\frac{2431 a^{18}}{17179869184}\left(\frac{d r_{2} d l_{2}}{d \phi d \phi}\right)\left(+\frac{77138650050 l_{2}^{16}}{r_{2}^{36}}-\frac{264475326850 l_{2}^{14}}{r_{2}^{34}}\right.$ $+\frac{364655436600 l_{2}^{12}}{r_{2}^{32}}-\frac{258787729200 l_{2}^{10}}{r_{2}^{30}}+\frac{100391791500 l_{2}^{8}}{r_{2}^{28}}$ $\left.-\frac{20822001200 l_{2}^{6}}{r_{2}{ }^{26}}+\frac{2082200120 l_{2}^{4}}{r_{2}{ }^{24}}-\frac{71597520 l_{2}^{2}}{r_{2}^{22}}+\frac{461890}{r_{2}^{20}}\right)$ 
NUMERICAL FACTORS
2. $\quad 0.375$
$3 . \quad 1.640625$
4. $\quad$ 5. 639648
5. $\quad 19.24530$
0.234375
6. $\quad 66.60249$
3. 076172
19. 24530
0. 1708984
4. 441223
98. 15103
43. 30193
0. 1345825
7. 234. 0337
457.8921
305. 2614
5. 773590
0. 1110306
80. 33195
7. 088113
8. 833.4602
2037.347
1832. 334
0. 0945082

\section{3001.926}
$8812.106 \quad 10027.57$
203. 4579
10. 10915. 34

37424.01
2946.372
51599. 77
294. 6372
744. 0746
132. 8705
$\begin{array}{ll}\text { 8. } 391819 & 0.0822727\end{array}$
$5570.871 \quad 1559.844$
9. 688472
0. 0728457
2946. 372

Equation 19 may appear formidable, but as with a similar formula in the 1930 paper (eq 11, p. 1266-67), it is comparatively simple in application. It contains the same system of vertical and diagonal moduli by which the literal parts of all terms after the second can be rapidly obtained, after which the terms are completed by applying the numerical factors.

The preceding discussion has been based upon the assumption that the center of the attracted segment is on the same level as the axes of the cylinders. While in practice this is nearly true for the balls, it is not the case with the elements of the beam, and in calculating the attraction on the beam, formulas 13 to 18 require a slight modification.

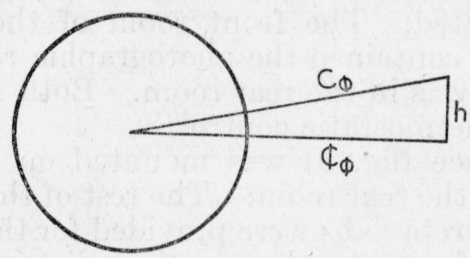

Figure 7.-Geometrical constants for attraction on beam.

In figure $7, C_{\phi}$ is the same as $C_{\phi}$ in eq 19 , the fundamental length which determines the potential of the cylinder at the center of the attracted element; $h$ is the elevation of the center of the element above the cylinder axis, $\phi_{\phi}$ is the horizontal projection of $C_{\phi}$, and $\dot{\phi}_{0}$ the horizontal projection of $C_{0}$. The slanting line $C_{\phi}$ is not directly measured, but is calculated from $\phi_{\phi}$ and $h$. The plane of the angle $\phi$ remains horizontal. The next step is to see what difference (if any) the introduction of $h$ will make in $C_{\phi}, r, l$, and their derivatives which occur in formula 19.

We have the fundamental equations:

$$
\left.\begin{array}{rl}
\phi_{\phi} & =\phi_{0}+R(1-\cos \phi), \text { whence } \frac{d \dot{\phi}}{d \phi}=R \sin \phi \text { and } \frac{d^{2} \dot{\phi}}{d \phi^{2}}=R \cos \phi \\
C_{\phi}^{2} & =\phi_{\phi}^{2}+h^{2} \\
r^{2} & =\phi_{\phi}^{2}+h^{2}+l^{2}
\end{array}\right\}
$$


For the derivatives of $C_{\phi}$, we have

$$
\left.\begin{array}{rl}
\frac{d C_{\phi}}{d \phi} & =\frac{\phi_{\phi}}{C_{\phi}} \frac{d \phi_{\phi}}{d \phi}=\frac{\phi_{\phi}}{C_{\phi}} R \sin \phi \\
\frac{d^{2} C_{\phi}}{d \phi^{2}} & =\frac{\phi_{\phi}}{C_{\phi}} \frac{d^{2} \phi_{\phi}}{d \phi^{2}}+\left(\frac{1}{C_{\phi}}-\frac{\phi_{\phi}^{2}}{C_{\phi}^{3}}\right)\left(\frac{d \phi_{\phi}}{d \phi}\right)^{2} \\
& =\frac{\phi_{\phi}}{C_{\phi}} R \cos \phi+\frac{h^{2}}{C_{\phi}^{3}} R^{2} \sin ^{2} \phi, \mid
\end{array}\right\}
$$

which assume still simpler values at the equilibrium angles.

The formulas for $l_{1}$ and $l_{2}$ and their derivatives, given by eq 15 and 16, are unaltered by the introduction of $h$. And this being the case, the formula for $r^{2}$ in eq 20 will give derivatives of the same form as those in eq 17, the only difference being that $\phi_{\phi}$ is written for $C_{\phi}$.

In the case of the attraction between cylinders and balls in the present work, the value of $h$ was always less than $1 \mathrm{~mm}$, and the minimum value of $\dot{\phi}$ about $13 \mathrm{~cm}$. This rendered the $h$-correction unnecessary for the balls, though obviously necessary for the beam.

\section{DESCRIPTION OF APPARATUS}

For the purpose of this work a special building was erected in a wooded section of the Bureau grounds, at a sufficient distance from parking lots and moving traffic to avoid disturbances. This building was 30 feet long and 20 feet wide, and was divided into two rooms by a partition. The walls of the building were of sheet steel with heat insulation, and were unprovided with windows. The inner partition was also heat-insulated. The front room of the building was the observing room, and contained the photographic recording apparatus. The torsion balance was in the rear room. Both rooms were electrically heated, with thermostatic control.

The torsion balance (fig. 8) was mounted on a massive concrete base in the center of the rear room. The rest of the floor was of wood, except that four concrete slabs were provided for the purpose of setting up a kathetometer for measuring vertical distances. The concrete base carried a circular steel plate $65 \mathrm{~cm}$ in diameter and $5 \mathrm{~cm}$ thick, at the center of which was the pendulum case, supported by three leveling screws. Three additional leveling screws were provided, which carried no load, but served to produce a more uniform gravitational field about the pendulum.

On the outer portion of the circular steel plate there was a circular steel ring $12 \mathrm{~cm}$ wide and $4 \mathrm{~cm}$ thick, resting on a complete ring of steel balls running in grooves. This steel ring carried the large attracting cylinders, and by rotating the ring the cylinders could be brought into the near or the far position as desired. To facilitate this adjustment the outer rim of the circular steel plate was provided with graduations and the rim of the circular ring carried a reference mark.

The cylinders were kept in place on the steel ring by four small aluminum wedges. As these wedges weighed about $0.21 \mathrm{~g}$ each, and the cylinders about $66,000 \mathrm{~g}$, no correction for the attraction of the wedges was necessary.

The pendulum case and its lid were made of soft iron, for magnetic shielding. Windows were provided for observing the elevation of the 


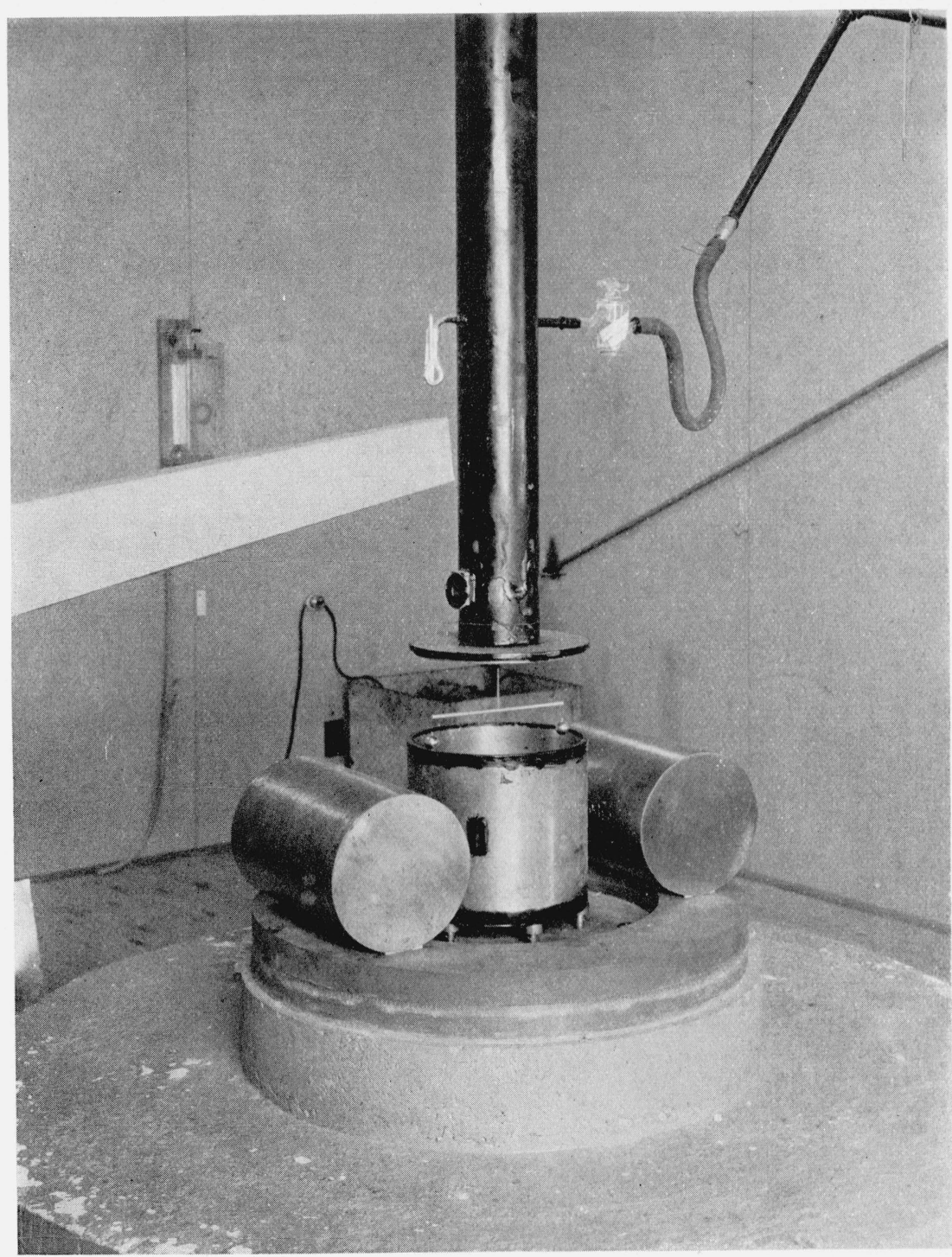

Figure 8.-Torsion balance. 
balls. The joint between the case and lid was sealed with Peixene wax. A brass tube attached to the lid carried the suspending filament. This tube was provided near the lower end with an observing window and four smaller windows for centering the filament. At the upper end the tube contained the movable torsion head described in the 1930 paper, by which the resting point of the filament could be adjusted from outside by means of a magnet without breaking the vacuum.

The beam of the pendulum with its attached balls can be seen in place in figure 8 . The beam was an aluminum tube $20 \mathrm{~cm}$ long and $0.5 \mathrm{~cm}$ in outside diameter. The beam was provided at its center with a vertical brass rod $5.5 \mathrm{~cm}$ long. A phosphor-bronze truss wire was run through the tube and its ends soldered to the upper end of the vertical brass rod. A piece of annealed tungsten filament, 0.0012 in. in diameter, was also run through the tube to provide a suspension for the balls, the centers of which were about $4 \mathrm{~cm}$ below the center of the beam. The balls were of platinum, provided with platinum hooks, and weighed about $87 \mathrm{~g}$ each. They were shaped to a spherical form by scraping through a steel die plate, as described in the 1930 paper.

The upper point of the beam truss was not joined directly to the suspending filament, but to a short piece of thin brass rod which carried a small optically flat silvered mirror at the level of the observing window. The connections with the filament at each end were made by small clamps.

The total mass of the beam was about $5.7 \mathrm{~g}$. The vacuum masses per unit length of the different parts were as follows, in grams per centimeter:

Aluminum tube 0.21254

Truss wire .00261

Filament supporting balls ..........

Two different tungsten filaments were used as a suspension for the pendulum. One of these was ordinary commercial lamp filament, hard-drawn, of diameter $0.0012 \mathrm{in}$. and tensile strength $288 \mathrm{~g}$. This filament had been coiled in the drawing process, and consequently exhibited considerable drift when first set up. The measurements with this filament were not begun until 3 months after setting up, by which time the drift of the resting point had been reduced to $0.04 \mathrm{~cm}$ in 15 hours, the photographic plate being $490 \mathrm{~cm}$ from the filament. The total load on the filament was about $182 \mathrm{~g}$.

After the completion of the measurements with this filament, we obtained, through the cooperation of W. E. Forsythe, of the General Electric Lamp Works at Cleveland, several pieces of specially annealed filament which had been kept straight in drawing and shipping. This filament, on account of being annealed, had a lower tensile strength than the hard-drawn filament, and a diameter of 0.0014 in. was necessary to give a tensile strength of $284 \mathrm{~g}$. This filament, although care was taken not to bend it in the process of setting up, showed a slight drift, which after 2 months' time was reduced to about half that of the hard-drawn filament.

The experience of Bearden ${ }^{2}$ of Johns Hopkins University with straight-drawn and especially annealed tungsten filament, in his work on the viscosity of air, led us to expect more precise results from the

\footnotetext{
${ }^{2}$ Phys. ${ }_{2}^{\circledR}$ Rev. 56 [2] 1023 (Nov. 15, 1939).
} 
annealed filament than from the hard-drawn one. The reverse, however, was the case. The average departure from the mean in our measurements with the hard-drawn filament was about half that obtained with the annealed filament, because of the fourth-power law of torsion. The times of swing with the annealed filament of diameter 0.0014 in. were about 1,880 and 1,640 sec in the far and near positions, respectively, with a difference of 240 sec. With the hard-drawn filament, of diameter 0.0012 in., the times of swing were increased to 2,920 and $2,200 \mathrm{sec}$, with a difference of $720 \mathrm{sec}$. This threefold increase in sensitivity with the hard-drawn filament apparently overbalanced any slight advantage of stability on the part of the annealed filament.

Of course, in geophysical surveying, where it is of importance to occupy as many stations as possible in the course of a week, it is out of the question to allow a month or more for a filament to stabilize itself; and in Bearden's work it was not necessary to determine the difference between two times of swing.

The vacuum used in the pendulum case was always less than $0.5 \mathrm{~mm}$. Experiments were made with a molecular pump giving a much lower pressure, but it was found that at pressures below $0.5 \mathrm{~mm}$ the damping factor of the pendulum became constant, indicating that at such pressures the air resistance was negligible compared to the internal friction in the filament.

\section{METHOD OF OBSERVING}

\section{ADJUSTMENTS}

(a) ADJUSTMENT OF CYLINDERS

The ideal adjustment of the cylinders requires that they be symmetrical with respect to a certain circle, which, of course, includes the condition of mutual parallelism. The tolerance for this adjustment is much greater than might be supposed. In all cases of asymmetry, one half of a cylinder will be a little nearer a ball than it should be and the other half a little too far away, and these two inaccuracies will partly cancel each other. For instance, calculation shows that, in the near position, a difference of $0.3 \mathrm{~mm}$ in the distance between the cylinders at their ends (28 cm apart) will affect only the seventh significant figure in the value of $d^{2} V / d \phi^{2}$, and for the far position the effect will be still less. It is, in fact, quite practicable to adjust for parallelism by a few trials to an accuracy of $0.1 \mathrm{~mm}$.

The cylinders were so accurately shaped that their lengths differed by only $0.01 \mathrm{~mm}$, and consequently it was easy to set them with their end faces in the same plane with an accuracy of $0.1 \mathrm{~mm}$ by laying a straightedge across the faces.

It was found also that the circular steel ring was sufficiently accurate to serve as the circle of reference for the cylinders and the pendulum. Measurements of the inside and outside diameters of the ring showed that no error greater than $0.2 \mathrm{~mm}$ existed in these dimensions. By means of the graduated circle around the rim of the steel plate it was possible to complete the adjustment of the cylinders for symmetry with respect to the circle. 


\section{(b) ADJUSTMENT OF PENDULUM}

For the pendulum three adjustments are necessary-azimuth, longitudinal, and transverse. These adjustments, as was found in the 1930 work, have a rather large tolerance, which in some cases, as in the azimuth setting in the near position, is increased by the changed position of the cylinders. Calculation gives the following errors in the result introduced by neglecting the errors in the separate adjustments in the near position. In the far position there will be still less effect in the result.

Transverse centering, error $0.5 \mathrm{~mm} \ldots 1$ part in 27,000

Longitudinal centering, error $0.5 \mathrm{~mm}_{---1}$ part in 26,000

Azimuth centering, error $0.1^{\circ} \ldots \ldots \ldots 1$ part in 65,000

The original adjustments for centering could be made to $0.1 \mathrm{~mm}$ and $0.1^{\circ}$. The centering adjustments were checked at intervals during the

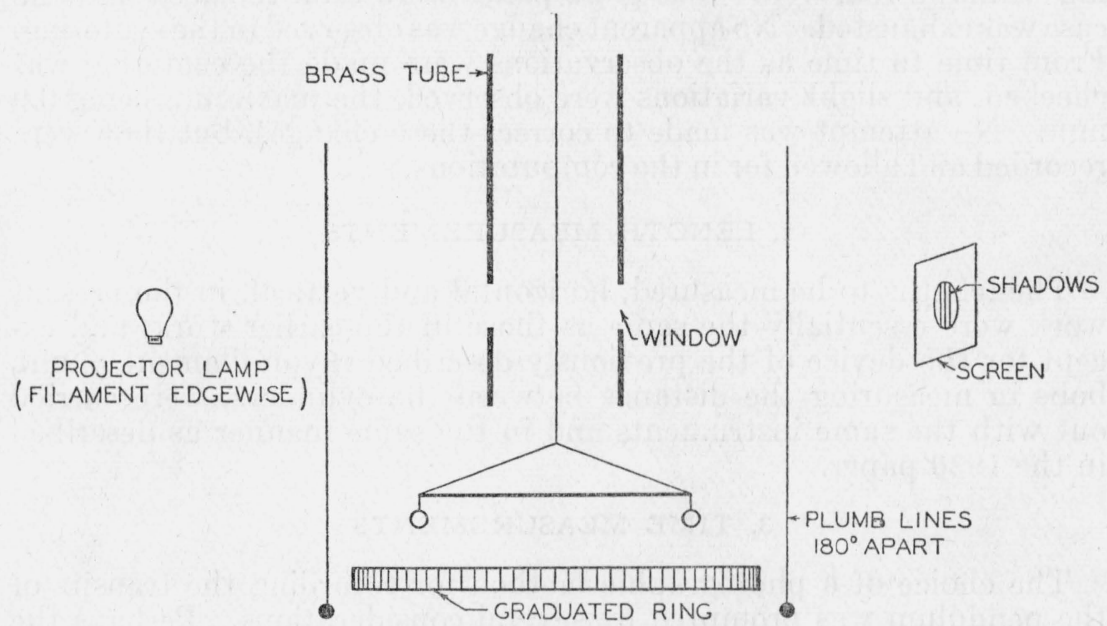

Figure 9.-Centering device.

work, and such errors as arose in the course of the work were recorded and allowed for in the computations.

The azimuth setting was made in the present work by direct observation. The lid of the pendulum case was lifted sufficiently to allow the beam to be held against two supports in such a position that the normal to the mirror passed approximately through the center of the recording apparatus, as determined by visual observation. Then by means of two plumb lines the azimuths of the centers of the ends of the beam were found to be $285.6^{\circ}$ and $107.2^{\circ}$, with a difference of $178.4^{\circ}$ instead of $180^{\circ}$. This showed that a transverse adjustment was necessary. Imagining such an adjustment to be made so that the azimuth at each end will be altered by $0.8^{\circ}$, the correct azimuth readings were found to be $286.4^{\circ}$ and $106.4^{\circ}$. Then, by turning the steel ring carrying the cylinders until the centers of the cylinders were at these azimuth readings, the cylinders could be set in the near position, and by turning $90^{\circ}$ farther, in the far position.

The pendulum case was then closed and the filament centered longitudinally and transversely, making use of the four observation 
windows in the tube carrying the filament (fig. 9). For this purpose two plumb bobs were hung close to the graduations on the circular steel plate, $180^{\circ}$ apart. A projector lamp was placed in line with these plumb bobs at a distance of about $3 \mathrm{~m}$ from the filament, giving approximately a point source of light, and a white cardboard screen was placed on the opposite side of the pendulum case, on which three sharp shadows could be obtained, two of the wire plumb lines and one of the filament. By moving the plumb lines around, always keeping them $180^{\circ}$ apart, their shadows could be made to coincide, and then by tilting the pendulum case by means of its leveling screws the shadow of the filament could be brought into coincidence with the shadow of the plumb lines. By carrying out this process in two positions $90^{\circ}$ apart, the filament could be centered with respect to the circular steel plate with an accuracy of $0.1 \mathrm{~mm}$.

This centering was made at first with the glass panes of the observing windows removed. The glass panes were then replaced and the case was exhausted. No apparent change was observed in the centering. From time to time as the observations were made the centering was checked, and slight variations were observed, the maximum being 0.9 $\mathrm{mm}$. No attempt was made to correct these changes, but they were recorded and allowed for in the computations.

\section{LENGTH MEASUREMENTS}

The lengths to be measured, horizontal and vertical, in the present work were essentially the same as those in the earlier work, and except for the device of the previously described rayon-filament plumb bobs in measuring the distance between the cylinders, were carried out with the same instruments and in the same manner as described in the 1930 paper.

\section{TIME MEASUREMENTS}

The choice of a photographic method for recording the transits of the pendulum was prompted by several considerations. Perhaps the most important of these was the desire to obtain an accurate record of transits at small amplitudes, as these have the greatest influence in the reduction of the time of swing to infinitesimal amplitude. Moreover, by making the method completely automatic, the necessity for the continuous presence of the observer was eliminated.

\section{(a) STANDARD OF TIME}

The standard of time employed was the primary frequency standard maintained by the radio laboratory of the National Bureau of Standards. This standard originates in a $100-\mathrm{kc}$ quartz oscillator, from which, by a submultiple generator, a 60 -cycle current was obtained. This standard 60-cycle current was amplified sufficiently to operate the signaling mechanism of the photographic recording apparatus. The frequency of the primary oscillator was constant to better than 1 part in 10 million.

The signals used in the recording apparatus were flashes of light at 5 -sec intervals. These were obtained by a revolving shutter in the form of a brass disk with radial slits cut in its periphery. This disk was rotated at a speed of one revolution a minute by means of 
a synchronous clock motor driven by the standard 60-cycle current. The disk carried 11 slits at intervals of $30^{\circ}$, the twelfth being omitted to mark the minutes.

\section{(b) OPTICAL SYSTEM}

Two slightly different optical systems were used. The first system, used with the hard-drawn filament, was an adaptation of a system previously used for visual observations. In this system the light from a 200-watt projector lamp was focused on a narrow slit close to the revolving disk. The light from the slit then entered the eyepiece of a telescope and was directed to the plane mirror carried by the torsion pendulum. After reflection at this mirror, the light was brought to a focus to form an image of the signaling slit on a photographic plate. As the pendulum swung, an image was recorded on the plate every 5 sec. The distance from mirror to plate was 490 $\mathrm{cm}$, and a deflection of $1^{\circ}$ at the mirror corresponded to a distance of $17 \mathrm{~cm}$ at the plate. The sharpness of the lines obtained was such that three lines per millimeter could easily be resolved by a low-power microscope.

For the measurements with the annealed filament the optical system was improved to give sharper images on the photographic plate (fig. 10). Here the light flashes from the revolving disk were focused on a stationary slit by means of an achromatic lens. It was found that the presence of this second slit gave a much sharper image on the plate. After passing through a concave lens of $25 \mathrm{~cm}$ focal length, the light fell on a convex lens of $400-\mathrm{cm}$ focal length mounted on the outside of the pendulum case just in front of the mirror carried by the pendulum. The light was reflected by the mirror back through the convex lens and was brought to a focus on the photographic plate. The sharpness of the lines obtained with this second system was such that five lines per millimeter could easily be resolved.

Except for the mirror on the pendulum and the convex lens on the outside of the pendulum case, the whole optical system, including the camera, was mounted on a concrete pier in the observing room (fig. 11). It was found that even though the apparatus and observing rooms were closed up and the temperature thermostatically controlled, there was sufficient turbulence caused by convection currents to be noticeable in the photographic record. For this reason a series of paper tubes was set up enclosing the light path in both directions. Undesirable reflections from the inner surfaces of these tubes, which would have fogged the plate, were prevented by a system of interior baffles.

A reference mark was provided on the photographic plate as a basis for measurements of the positions of the recorded images. This mark was the shadow of a fine wire fastened to the front panel of the camera (fig. 12). The shadow was made by the light from a neon lamp behind a pinhole in the optical path about $200 \mathrm{~cm}$ in front of the camera. The plate being continuously illuminated by the faint red light was fogged just enough to show the shadow of the wire as a white line, without interfering perceptibly with the definition of the recorded images.

(c) THE CAMERA

The camera consisted of a metal box in which the plateholder could move only in a vertical direction (fig. 12). A spring on the 
26 Journal of Research of the National Bureau of Standards

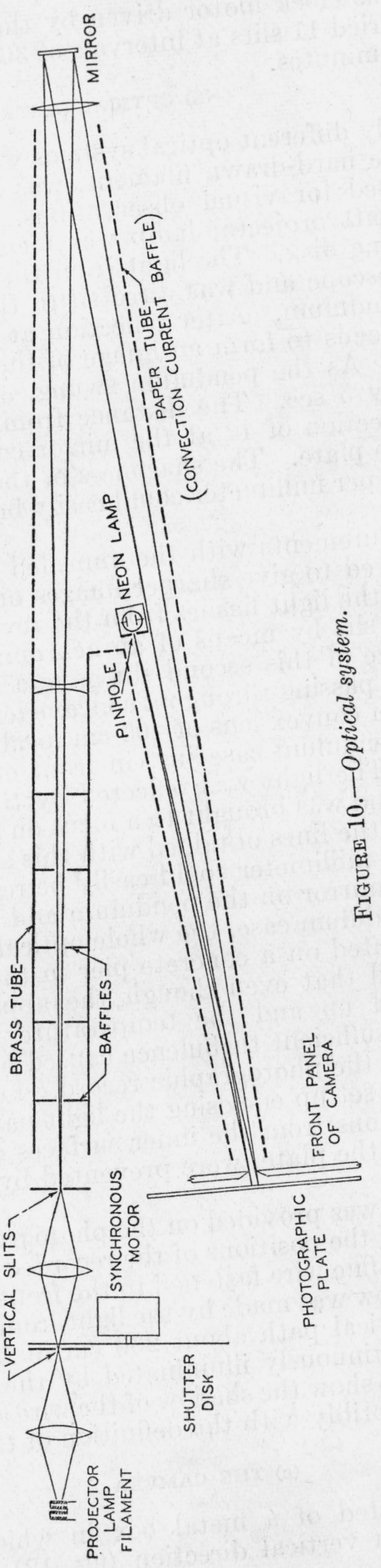




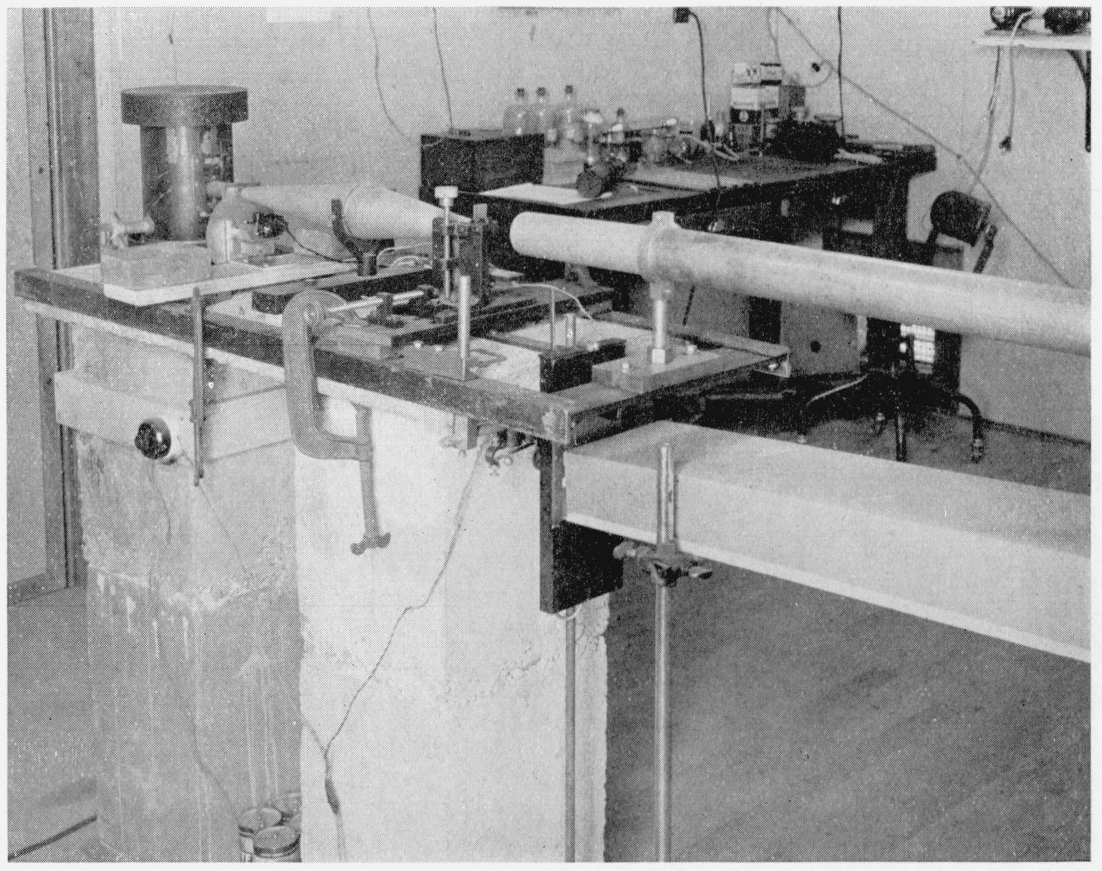

Figure 11.-Photographic recording apparatus. 
plateholder bearing against the side of the camera prevented any sidewise motion of the plateholder. The beam of light reflected from the moving mirror entered through a horizontal slot cut through the front panel of the camera. With the pendulum at rest, the image of the illuminated slit was centered vertically across this slot and the motion of the mirror made the beam traverse the length of the slot. Thus, only as much of the plate was exposed during a given transit as was exposed by the slot during the transit. A guillotine safety

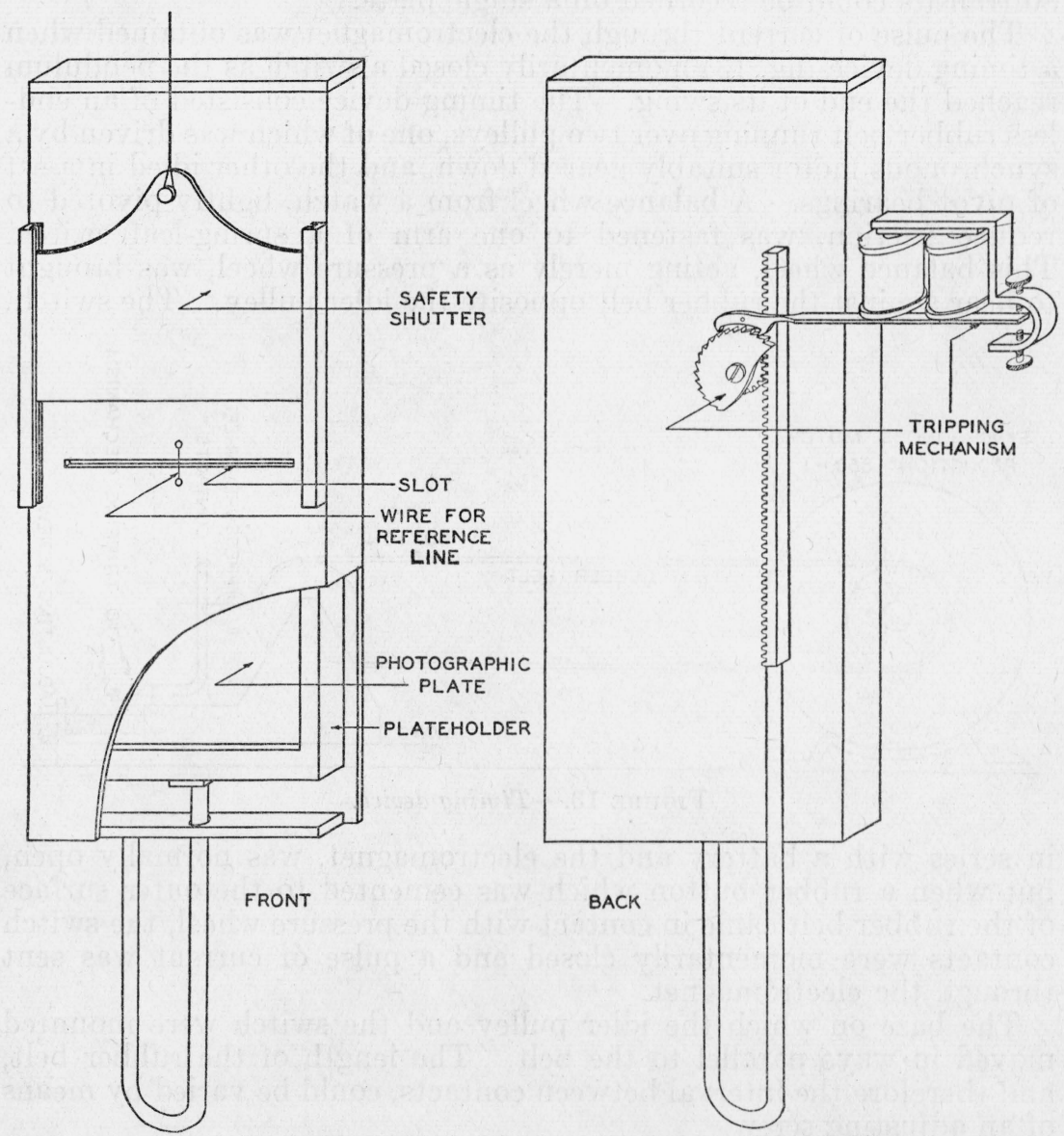

Figure 12.-Camera and tripping mechanism.

shutter on the front panel could be dropped to cover the slot whenever it was desired to make the camera light-tight.

Between transits, the vertical position of the plateholder was changed automatically by means of a tripping mechanism mounted on the back of the camera (fig. 12). Essentially, the mechanism consisted of a pawl-and-ratchet escapement. The pawl was actuated by an electromagnet. The ratchet was rigidly connected to a pinion which in turn was geared to a rack sliding in ways. The rack formed one prong of a fork whose other prong entered the camera through a 
hole in its bottom. The plateholder rested on the end of the prong within the camera. Thus, when a pulse of current from a timing device actuated the electromagnet, the escapement permitted the plateholder to drop by gravity through a predetermined distance.

The camera accommodated 4 - by 5 -in. photographic plates. The choice of this particular size was dictated by the availability of a traveling microscope whose screw was $10 \mathrm{~cm}$ in length. The plate emulsion was of the modern high-speed panchromatic variety. Over 80 transits could be recorded on a single plate.

The pulse of current through the electromagnet was obtained when a timing device (fig. 13) momentarily closed a switch as the pendulum reached the end of its swing. The timing device consisted of an endless rubber belt running over two pulleys, one of which was driven by a synchronous motor suitably geared down, and the other idled in a set of pivot bearings. A balance wheel from a watch, lightly pivoted to reduce friction, was fastened to one arm of a spring-leaf switch. This balance wheel, acting merely as a pressure wheel, was brought to bear against the rubber belt opposite the idler pulley. The switch,

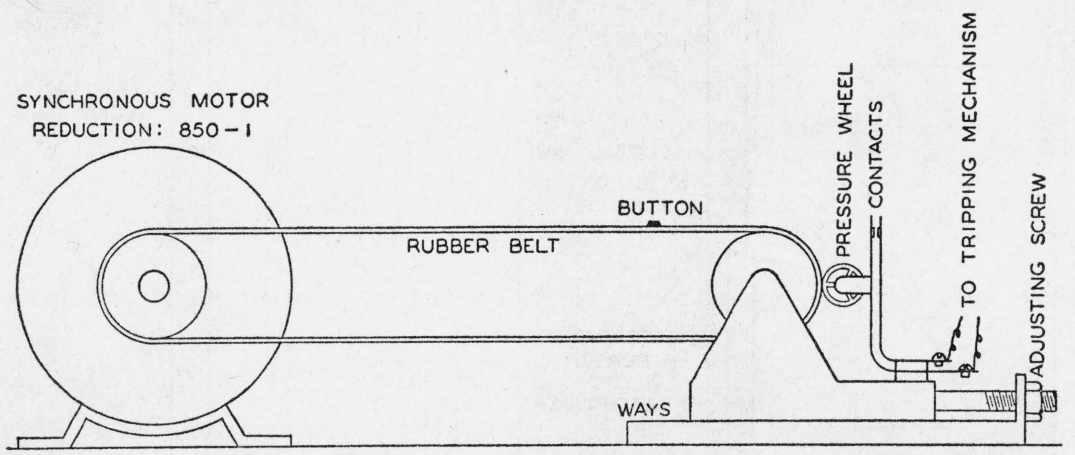

FIGURE 13.-Timing device.

in series with a battery and the electromagnet, was normally open, but when a rubber button which was cemented to the outer surface of the rubber belt came in contact with the pressure wheel, the switch contacts were momentarily closed and a pulse of current was sent through the electromagnet.

The base on which the idler pulley and the switch were mounted moved in ways parallel to the belt. The length of the rubber belt, and therefore the interval between contacts, could be varied by means of an adjusting screw.

A different driving pulley was used with each type of swing, since the difference in the half period in the "near" and "far" positions was more than could be taken care of by the adjusting screw.

Using a pulley of the proper size, the length of the rubber belt was adjusted so that the interval between contacts was very nearly equal to the mean half period of the pendulum. The button on the rubber belt was brought into position to make contact, and the motor was started as the pendulum reached the end of its swing. Then throughout a series of 60 to 70 transits the time of contact and the time the pendulum reached the end of its swing remained very nearly synchronized. 
Records were made at night, from five o'clock in the afternoon until eight 0 'clock the next morning. The pendulum was started swinging (by movable weights outside the case, as in the 1930 paper) and the plateholder adjusted in the camera. The guillotine safety shutter was dropped and the room darkened. The operator then pulled the slide of the plateholder, and going outside of the house and closing the door, pulled a wire which lifted the guillotine shutter, and the run was on. This process was reversed the next morning, the safety shutter being dropped before the door was opened.

The precision obtainable by this method of photographic recording can be seen from the following examples (table 1), taken from observations in the near position with the annealed filament. Measurement of the photographic record by a traveling microscope gave the following figures, for two transits, one near the beginning and one near the end of a 14-hour run, the central reference line on the record being taken as $50 \mathrm{~mm}$.

TABLE 1.-Time-scale relation

TRANSIT 5

TRANSIT 66

\begin{tabular}{|c|c|c|c|c|c|}
\hline Time & $\begin{array}{l}\text { Scale } \\
\text { reading }\end{array}$ & $\begin{array}{c}\text { Scale } \\
\text { difference }\end{array}$ & Time & $\begin{array}{l}\text { Scale } \\
\text { reading }\end{array}$ & $\begin{array}{c}\text { Scale } \\
\text { difference }\end{array}$ \\
\hline $\begin{array}{c}\text { sec } \\
40\end{array}$ & $\begin{array}{c}m m \\
15.92\end{array}$ & \multirow{12}{*}{$\begin{array}{l}m m \\
6.33 \\
6.34 \\
6.33 \\
6.38 \\
6.36 \\
6.38 \\
6.38 \\
6.34 \times 2 \\
6.28\end{array}$} & $\begin{array}{l}\mathrm{sec} \\
30\end{array}$ & $\begin{array}{c}m m \\
44.33\end{array}$ & \\
\hline 35 & 22.25 & & 35 & 44. 90 & 0.57 \\
\hline 30 & 28.59 & & 40 & 45.48 & \\
\hline 25 & 34.92 & & 45 & 46. 06 & \\
\hline 20 & 41.30 & & 50 & 46.61 & \\
\hline 15 & 47.66 & & 55 & 47.17 & $57 \times 2$ \\
\hline 10 & 54.04 & & 5 & 48.31 & .56 \\
\hline 5 & 60.42 & & 10 & 48.87 & .58 \\
\hline 55 & 73.10 & & 15 & 49.45 & 57 \\
\hline \multirow[t]{3}{*}{50} & 79.38 & & 20 & 50.02 & \\
\hline & & & 25 & 50.57 & \\
\hline & & & 30 & 51.14 & .08 \\
\hline
\end{tabular}

It will be seen that although the speeds in these transits differ by a factor of 11, the speed in each transit is practically uniform within the space covered by each record, and interpolation is safe. By interpolation we next obtain for each transit of the run a table with integral values of the scale readings and nonintegral values of the time, such as would be obtained by chronographic recording of visual observations.

It was found that with 60 or more transits in each record, considerable time could be saved by making a change in the procedure of the 1930 paper. The calculation was carried out in the usual way up to the point where the mean midpoint of the swing was determined as the average of five separate values. The next step, the sinusoidal corrections, took a great deal of time, and it was found that this could be avoided. 
The alternative procedure adopted can be illustrated by an example. Taking transit 5 , we obtain by the aid of transits 4 and 6 the following midpoints on the scale in millimeters:

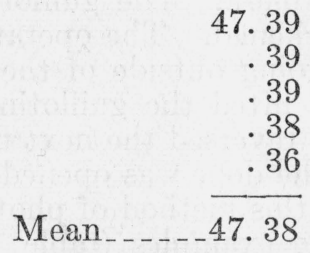

From the time-scale relation for this transit (table 1), we find by interpolation the following values of the time at the mean midpoint:

$28 \min 15.22 \mathrm{sec}$.

\begin{tabular}{c}
$28 \min 15.22$ sec. \\
.23 \\
.23 \\
.23 \\
.21 \\
\hline Mean__._28 min 15. 22 sec.
\end{tabular}

The corresponding value for transit 7 is found to be 55 min 35.45 sec, and the difference of these times, $27 \mathrm{~min} 20.23 \mathrm{sec}$, gives us one value for the damped period of the pendulum. From this point the procedure is the same as that in the 1930 paper.

\section{RESULTS}

The computations for the present work are much too extensive to be given here. The complete computations and original notebooks are preserved in the Library at the National Bureau of Standards. As an illustration of the general procedure, we may summarize the calculation of one result in table 2.

\section{TABLE 2.-Calculation of $G$}

[Hard-drawn filament]

Far position, May 15-18, 1940. Periodic time $=T_{F}=2924.20$ sec. Temp. $24.1^{\circ} \mathrm{C}$.

$I=$ moment of inertia $=17735.4$

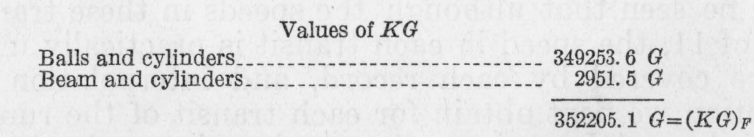

Near position, May 22-24, 1940. $T_{N}=2200.12 \mathrm{sec}$. Temp. $24.1^{\circ} \mathrm{C}$.

$I=17735.4$

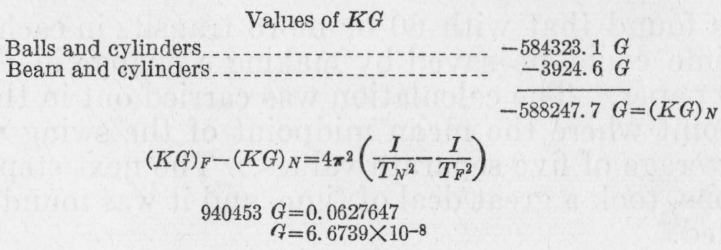


Five values of $G$ were obtained with each filament, hard-drawn and annealed. These values are given in table 3 , with their average departures from the mean.

TABLE 3.-Values of $G$

\begin{tabular}{|c|c|c|c|}
\hline \multicolumn{2}{|c|}{ Hard-drawn filament } & \multicolumn{2}{|c|}{ Annealed filament } \\
\hline $\begin{array}{r}6.6739 \\
56 \\
69 \\
62 \\
51\end{array}$ & $\begin{array}{r}\Delta \\
0.0016 \\
1 \\
14 \\
7 \\
4\end{array}$ & $\begin{array}{r}6.6670 \\
667 \\
703 \\
707 \\
680\end{array}$ & 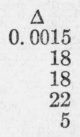 \\
\hline 6.6755 & 0.0008 & 6. 6685 & 0.0016 \\
\hline
\end{tabular}

It will be seen that the precision of the results with the hard-drawn filament is twice that of the results with the annealed filament. Weighting the results in this proportion, we obtain for the weighted mean:

$$
6.6732 \pm 0.0031 .
$$

In view of the fact that the average departure from the mean appears in the third decimal place, the fourth decimal place may be dropped, and we obtain for the final result:

$$
6.673 \pm 0.003 \times 10^{-8} \mathrm{~cm}^{3} \mathrm{~g}^{-1} \mathrm{sec}^{2}
$$

The final result in the 1930 paper was:

$$
6.670 \pm 0.005 \times 10^{-8} \mathrm{~cm}^{3} \mathrm{~g}^{-1} \mathrm{sec}^{-2} \text {. }
$$

These two results agree well with each other, but it is to be noted that the increase in precision in the present work is hardly appreciable. It will also be noted that the present results show the same peculiarity of pattern as did those in the 1930 paper. The results with the hard-drawn filament differ from those with the annealed filament by an amount greater than can be accounted for by the departures from the mean.

It is difficult to explain this anomaly. The only point of difference in the two series of measurements was the difference in the filament, and while irregular variations in the torsion constant of the filament are to be expected, and would account for random variations in the individual results with any one filament, what is needed to account for the observed anomaly in the results with the two filaments is a regular variation of such a nature as to be incredible.

Comparing the 1930 results with those of the present work, the outstanding feature is that a carefully planned and executed attempt to increase the precision of the 1930 results has met with but slight success. The conclusion may be drawn that the limit of the possibilities of the torsion balance has been reached.

Washington, March 30, 1942. 